\title{
A Micro-Mechanical Model for the Homogenisation of Masonry
}

\author{
A. ZUCCHINI $^{1}$ \\ ENEA, INN.FIS.MACO, C.R.E. “E.Clementel”, v.Don Fiammell,2, I - 40129 Bologna, Italy.
}

\section{P.B. LOURENÇO *}

University of Minho, Department of Civil Engineering, Azurém, P - 4800-058 Guimarães, Portugal

\footnotetext{
${ }^{1}$ E-mail: zucchini@indy.bologna.enea.it,Ph: +39 0516098256, Fax: +390516098062

*E-mail: pbl@eng.uminho.pt, Ph: + 351253 510200, Fax: + 351253510217
} 


\section{ABSTRACT}

Masonry is a composite material made of units (brick, blocks, etc.) and mortar. For periodic arrangements of the units, the homogenisation techniques represent a powerful tool for structural analysis. The main problem pending is the errors introduced in the homogenisation process when large difference in stiffness are expected for the two components. This issue is obvious in the case of non-linear analysis, where the tangent stiffness of one component or the tangent stiffness of the two components tends to zero with increasing inelastic behaviour.

The paper itself does not concentrate on the issue of non-linear homogenisation. But as the accuracy of the model is assessed for an increasing ratio between the stiffness of the two components, the benefits of adopting the proposed method for non-linear analysis are demonstrated. Therefore, the proposed model represents a major step in the application of homogenisation techniques for masonry structures.

The micro-mechanical model presented has been derived from the actual deformations of the basic cell and includes additional internal deformation modes, with regard to the standard two-step homogenisation procedure. These mechanisms, which result from the staggered alignment of the units in the composite, are of capital importance for the global response. For the proposed model, it is shown that, up to a stiffness ratio of one thousand, the maximum error in the calculation of the homogenised Young's moduli is lower than five percent. It is also shown that the anisotropic failure surface obtained from the homogenised model seems to represent well experimental results available in the literature.

\section{KEYWORDS}

Composites, numerical techniques, homogenisation techniques, masonry 


\section{INTRODUCTION}

Masonry is a composite material made of units and mortar, normally arranged periodically. Utilising the material parameters obtained from experiments and the actual geometry of both components, viz. units (e.g. bricks, blocks or stones) and joints, it is possible to numerically reproduce the behaviour of masonry structures, see e.g. Lofti and Shing (1994), and Lourenço and Rots (1997). Nevertheless, the representation of each unit and each joint becomes impractical in case of real masonry structures comprising a large number of units.

The alternative is to describe the composite behaviour of masonry in terms of macro or average stresses and strains so that the material can be assumed homogeneous. This problem can be approached, basically, from two directions. A possible direction is to gather extensive experimental data that can be used confidently in the analyses. It is stressed that the results are limited to the conditions under which the data are obtained. New materials and/or application of a well known material in different loading conditions might require a different set of costly experimental programs. Another direction, adopted in this paper, is to seek a more fundamental approach which resorts to homogenisation techniques. This approach, which aims at describing the behaviour of the composite from the geometry and behaviour of the representative volume element (or basic cell, see Fig. 1), grants us a predictive capability.

The techniques of homogenisation, Bakhvalov and Panasenko (1989),

are currently becoming increasingly popular among the masonry 
community. A method that would permit to establish constitutive relations in terms of averaged stresses and strains from the geometry and constitutive relations of the individual components would represent a major step forward in masonry modelling. Given the difficult geometry of the masonry basic cell, a close-form solution of the homogenisation problem seems to be impossible, which leads , basically, to three different lines of action.

The first, very powerful approach is to handle the brickwork structure of masonry by considering the salient features of the discontinuum within the framework of a generalised / Cosserat continuum theory. This elegant and efficient solution, Besdo (1985) and Mühlhaus (1993), possesses some inherent mathematical complexity and has not been adopted by many researchers, even though being capable of handling the unit-mortar interface and true discontinuum behaviour. The step towards the practical application of such an approach is still to be done.

A second approach, Anthoine (1995,1997) and Urbanski et al. (1995), is to apply rigorously the homogenisation theory for periodic media to the basic cell, i.e. to carry out a single step homogenisation, with adequate boundary conditions and exact geometry. It is stressed that the unit-mortar interface has not yet been accounted for by researchers. The complexity of the masonry basic cell implies a numerical solution of the problem, which has been obtained using the finite element method. The theory was thus used by the cited authors to determine macro-parameters of masonry and not, actually, to carry out analysis at the structural level. In fact, the rigorous application of the homogenisation theory for the non-linear behaviour of the complex masonry basic cell implies solving the problem for all possible 
macroscopic loading histories, since the superposition principle does not apply anymore. Thus, the complete determination of the homogenized constitutive law would require an infinite number of computations.

The third approach can be considered as an "engineering approach”1, aiming at substituting the complex geometry of the basic cell by a simplified geometry so that a close-form solution of the homogenisation problem is possible. Keeping in mind the objective of performing analysis at the structural level, Pande et al. (1989), Maier et al. (1991) and Pietruszczak and Niu (1992) introduced homogenisation techniques in an approximate manner. The homogenisation has generally been performed in two steps, head (or vertical) and bed (or horizontal) joints being introduced successively. In this case masonry can be assumed to be a layered material, which simplifies the problem significantly. Lourenço (1996) further developed the procedure, presenting a novel matrix formulation that allows a much clearer implementation of linear elastic homogenisation algorithms and also a relatively simple extension to nonlinear behaviour. Again, it is stressed that the unit-mortar interface has not been accounted for by the cited researchers.

The use of two separate homogenisation steps does not explicitly account for the regular offset of vertical mortar joints belonging to two consecutive layered unit courses. Moreover, the final result depends on the order in which the two homogenisation processes are carried out.

\footnotetext{
1 "Engineering" is used here not in the sense that it is empiric or practical but in the sense that must be engineered from reasoning.
} 
Nevertheless, this simplified homogenisation approach has been used by several authors and performs very satisfactorily in the case of linear elastic analysis, Anthoine (1995) and Lourenço (1997). For the case of non-linear analysis, where the ratio between the stiffness of unit and mortar becomes larger, the simplified homogenisation approach leads to non-acceptable errors and should not be used. Lourenço (1997) has shown that large errors can occur in the standard two step homogenisation technique if there are large differences of stiffness $(>10)$ between unit and mortar. Anthoine $(1995,1997)$ has shown that the standard two step homogenisation technique does not take into account the arrangement of the units in the sense that different bond patterns (running bond and stack bond for example) may lead to exactly the same result.

A different engineering approach has been proposed by Bati et al. (1999), in which a close-form solution of the periodic arrangement of units and mortar has been obtained, by substituting the parallelepiped-shaped units by elliptic cylinders. This mathematically elegant solution does not represent well the geometry and it is unclear if it represents an advantage with regard to the standard two-step homogenisation technique.

The present paper presents a new micro-mechanical model, for masonry in stretcher bond ${ }^{2}$, to overcome the limitations of the standard twostep homogenisation by a more detailed simulation of the interactions

2 "Stretcher bond" represents the typical arrangement of masonry units in a wall, with an offset half unit for the vertical mortar joints belonging to two consecutive masonry courses. The "stacked bond" arrangement, in which the vertical joints run continously through all the courses, is not allowed for structural purposes by most masonry codes. 
between the different internal components of the basic cell. The model can still be considered as an engineering approach, in which an ingenious

observation of the behaviour of masonry leads to the simulation of additional internal deformation mechanisms of the joints, that become more and more important for increasing unit/mortar stiffness ratios. At this stage, the unit-mortar interface is not considered in the model.

It is noted that micro-mechanical approaches that consider additional internal deformation mechanisms have been derived independently by van der Pluijm (1999) for the analysis of masonry subjected to flexural bending and by Lopez et al. (1999) for the non-linear analysis of masonry walls subjected to in-plane loading.

In this paper, the full three-dimensional behaviour will be considered and attention will be given to a comparison between the results from a detailed finite element analysis (FEA) and the proposed micro-mechanical homogenisation model, in order to demonstrate the efficiency of the proposed solution. Finally, the adequacy of the model to reproduce the anisotropic failure surface of masonry will be discussed by means of a comparison with available experimental results.

\section{Descriptive analysis of masonry}

As a consequence of the differences in stiffness between units and mortar, a complex interaction between the two masonry components occurs when masonry is deformed. The differences in stiffness cause a unequal 
distribution of deformations over units and mortar, compared with the average deformation of masonry composite. As a result the individual (internal) stresses of units and mortar deviate from the average (external) stresses of the composite.

For the purpose of understanding the internal deformational behaviour of masonry components (units and mortar), when average deformations occur on the boundaries of the basic cell, detailed finite element calculations have been carried out for different loading conditions. For a clear discussion of the internal distribution of stresses, a right-oriented $x-y-z$ coordinate system was defined, where the $x$-axis is the parallel to the bed joints, the $y$ axis is parallel to the head joints and the z-axis is normal to the masonry plane, see Fig. 2. This figure also shows the components considered in the present paper. The cross joint is defined as the mortar piece of the bed joint that is connected to the head joint.

The mesh used in the analyses is depicted in Fig. 3 and consists of $24 \times 4 \times 12$ twenty-noded quadratic 3-D elements with reduced integration. The unit dimensions are $210 \times 100 \times 52 \mathrm{~mm}^{3}$ and the mortar thickness is 10 $\mathrm{mm}$. The assumption that the units are stiffer than the joints is usually made by the masonry research community. In the present analysis, in order to better understand the deformational behaviour of the mortar, the units are considered infinitely stiff (for this purpose, the adopted ratio between unit and mortar stiffness was 1000).

Fig. 4 illustrates the deformation corresponding to the analysis of the basic cell under compression along the axes $x, y$ and $z$, and under shear in the planes $x y, x z$ and $y z$. Loading is applied with adequate tying of the nodes 
in the boundaries, making use of the symmetry and antisymmetry conditions appropriate to each load case. Therefore, the resulting loading might not be associated with uniform stress conditions or uniform strain conditions. Linear elastic behaviour is assumed in all cases.

Fig. 4a demonstrates that, for compression along the $x$-axis, the unit and the bed joint are mostly subjected to normal stresses, the bed joint is strongly distorted in shear and the cross joint is subjected to a mixed shear / normal stress action. While the cross joint effect can be neglected if the cross joint is small compared to the basic cell, the shear of the bed joint must be included in the micro-mechanical model of masonry for stiff units.

Fig. 4b demonstrates that, for compression along the $y$-axis, the unit and the bed joint are mostly subjected to normal stresses, and the head and cross joints are subjected to a mixed shear / normal stress action. These relatively local effects are difficult to include in the micro-mechanical model, have small influence on the overall behaviour of the basic cell and will not be considered. This is confirmed by the results of Lourenço (1997) where it was shown that the standard two-step homogenisation technique, which neglects such effects, leads to almost exact results (errors smaller than $2 \%$ for ratios unit / mortar stiffness up to 1000).

Fig. 4c demonstrates that, for compression along the $z$-axis, all components of the basic cell are subjected to a truly homogeneous state of normal stress. This again is confirmed by the results of Lourenço (1997) where it was shown that the standard two-step homogenisation technique leads to almost exact results (errors smaller than $0.2 \%$ for ratios unit / mortar stiffness up to 1000). 
Fig. 4d demonstrates that, for $x y$ shear, the unit and the head joint are mostly subjected to shear stresses, the bed joint is strongly distorted in the normal direction (tension) and the cross joint is subjected to a mixed shear / normal stress. Due to antisymmetric conditions, the neighbouring basic cells will feature normal compression in the bed joint. While the cross joint effect can be neglected if the cross joint is small compared to the basic cell, the normal stress of the bed joint must be included in the micro-mechanical model.

The deformation of the basic cell under $x z$ shear is shown in Fig.4e. The cell components are mostly subjected to shear stresses, with unit and head joint deformed in the horizontal plane, while the bed joint is distorted also in the vertical plane. Therefore the shear stress $\sigma_{y z}$ cannot be neglected in a micro-mechanical model.

Finally, the deformation of the basic cell under $y z$ shear is shown in Fig.4f. All cell components are mainly distorted by shear in the vertical plane, while minor local stress components do not produce significant overall effects.

\section{Formulation of the micro-mechanical model}

\subsection{General}

Lourenço (1997) has shown that large errors can occur in the standard two-step homogenisation technique if there are large differences of stiffness ( $>10)$ between unit and mortar. The micro-mechanical model presented in 
this paper overcomes this limitation by a more detailed simulation of the interactions between the different internal components of the basic cell.

The main idea of this approach, derived from observations of deformations calculated with the finite element analyses shown in the previous section, is that the standard two-step homogenisation technique neglects some deformation mechanisms of the bed joint, that become more and more important for increasing unit/mortar stiffness ratios, such as:

- vertical normal stress in the bed joint, when the basic cell is loaded with in-plane shear;

- in-plane shear of the bed joint, when the basic cell is loaded with an horizontal in-plane normal stress;

- out-of-plane shear $\sigma_{y z}$ of the bed joint, when the basic cell is loaded with out-of-plane shear stress $\sigma_{x z}$.

These mechanisms are due to the staggered alignment of the units in a masonry wall and are neglected by the standard two-step homogenisation techniques, which are based on the assumption of continuous perpendicular head joints.

Due to the superposition principle, which applies in linear problems, the elastic response of the basic cell to a generic load can be determined by studying six basic loading conditions: three cases of normal stress and three cases of simple shear. In the present formulation, for each loading case and each basic cell component, suitably chosen components of the stress and strain tensors are assumed to be of relevance for the stress-strain state of the basic cell, while all the others are neglected, see Fig. 6 and Fig. 8 for 
examples. Equilibrium is, of course, ensured for all loading cases. The number of unknowns of the problem is larger than in the usual homogenisation procedure in order to take into account the above "second order" effects. The unknown internal stresses and strains can be found from equilibrium equations at internal interfaces between basic cell components, with a few ingenious assumptions on the cross joint behaviour and on the kinematics of the basic cell deformation, see Fig. 5 for the adopted geometric symbols. The equivalent properties of an homogenised material are then easily derived from the internal stresses and strains, by forcing the macro-deformation of the model and of the homogenised material to be the same, meaning that both systems must contain the same strain energy.

\subsection{Young's moduli and Poisson's coefficients}

The Young's moduli and the Poisson's coefficient of an equivalent orthotropic material can be derived from the elastic strains of the basic cell loaded with a uniform normal stress on the two faces perpendicular to a given axis $(x, y$ or $z)$. All other stresses vanish on the boundary. Fig. 9 shows the case of uniform loading in the horizontal in-plane direction ( $x$-axis). In this case all shear stresses and strains inside the basic cell are neglected, except the in-plane shear stress and strain $\left(\sigma_{x y}\right.$ and $\left.\varepsilon_{x y}\right)$ in the bed joint and in the unit. Non-zero stresses and strains are assumed to be constant in each basic cell component, except the normal stress $\sigma_{x x}$ in the unit, which must be a linear function of $x$ to account for the effect of the shear $\sigma_{x y}$ in the bed joint, and the shear stress $\sigma_{x y}$ in the unit, linear in $\mathrm{y}$. 
With these hypotheses, the following relations hold for the stresses at internal or boundary interfaces:

(1) Interface brick - head joint $\quad \sigma_{x x}^{2}=\bar{\sigma}_{x x}^{b}-\sigma_{x y}^{1} \frac{l-t}{2 h}$

(2) Interface brick - bed joints $\quad \sigma_{y y}^{b}=\sigma_{y y}^{1}$

(3) Right boundary $\quad h \sigma_{x x}^{2}+2 t \sigma_{x x}^{3}+h\left(\bar{\sigma}_{x x}^{b}+\sigma_{x y}^{1} \frac{l-t}{2 h}\right)=2(h+t) \sigma_{x x}^{0}$

(4) Upper boundary $\quad l \sigma_{y y}^{b}+t \sigma_{y y}^{2}=(l+t) \sigma_{y y}^{0}$

(5) Front boundary $2 t h \sigma_{z z}^{2}+2(l-t) t \sigma_{z z}^{1}+2 l h \sigma_{z z}^{b}+4 t^{2} \sigma_{z z}^{3}=[2 t h+2(l+t) t+2 l h] \sigma_{z z}^{0}$

and for the strains:

(6) Upper boundary

$$
\begin{gathered}
2 t \varepsilon_{y y}^{1}+h \varepsilon_{y y}^{b}=h \varepsilon_{y y}^{2}+2 t \varepsilon_{y y}^{3} \\
t \varepsilon_{x x}^{2}+l \bar{\varepsilon}_{x x}^{b}=2 t \varepsilon_{x x}^{3}+(l-t) \varepsilon_{x x}^{1} \\
\varepsilon_{z z}^{b}=\varepsilon_{z z}^{1} \\
\varepsilon_{z z}^{b}=\varepsilon_{z z}^{2}
\end{gathered}
$$

(8) Front boundary

(9) Front boundary

where $l$ is half of the unit length, $h$ is half of the unit height and $t$ is half of the bed joint width. Unit, bed joint, head joint and cross joint variables are indicated throughout this paper respectively by the superscripts $b, 1,2$ and 3, respectively. $\bar{\sigma}_{x x}^{b}$ and $\bar{\varepsilon}_{x x}^{b}$ are the mean value of the normal stress $\sigma_{x x}$ and normal strain $\varepsilon_{x x}$ in the unit. $\sigma_{x x}^{0}, \sigma_{y y}^{0}, \sigma_{z z}^{0}$ are the uniform normal (macro) stresses on the faces of the homogenised basic cell, respectively in the $x$-, $y$ - and $z$-direction. The equilibrium of the unit (Fig. 7) yields:

$$
h \sigma_{x x}^{b 1}+(l-t) \sigma_{x y}^{1}=h \sigma_{x x}^{b 2}
$$

where we assume that the shear acts only on the bed-unit interface (l-t). 
If $\sigma_{x x}^{b}$ is linear in $x$, its mean value in the mid-unit (equal to the mean value in the unit) is:

$$
\bar{\sigma}_{\mathrm{xx}}^{\mathrm{b}}=\frac{\sigma_{\mathrm{xx}}^{\mathrm{b} 1}+\sigma_{\mathrm{xx}}^{\mathrm{b} 2}}{2}
$$

From Eqs.(10,11) we get :

$$
\begin{aligned}
& \sigma_{x x}^{b 1}=\bar{\sigma}_{x x}^{b}-\sigma_{x y}^{1} \frac{1-t}{2 h} \\
& \sigma_{x x}^{b 2}=\bar{\sigma}_{x x}^{b}+\sigma_{x y}^{1} \frac{1-t}{2 h}
\end{aligned}
$$

which have been used in Eqs. $(1,3)$. The couple required for the momentum equilibrium of one fourth of the unit in the basic cell (Fig. 6) derives from the neighbouring cell along y-axis. The symmetric unit quarter of the cell above (Fig. 7) reacts at the centre line of the unit with a couple due to a selfequilibrating vertical stress distribution, which is neglected in the model.

In Eqs.(1-9) the unknown stresses and strains in the cross joint can be eliminated by means of the following relations:

$$
\begin{gathered}
\varepsilon_{y y}^{3}=\frac{E_{2}}{E_{3}} \varepsilon_{y y}^{2} \quad \sigma_{z z}^{3}=\frac{E_{3}}{E_{1}} \sigma_{z z}^{1} \quad \varepsilon_{x x}^{3}=\frac{E_{1}}{E_{3}} \varepsilon_{x x}^{1} \\
\sigma_{x x}^{3}=\sigma_{x x}^{1}
\end{gathered}
$$

Eqs.(13) assume that the cross joint behaves as a spring connected in series with the bed joint in the $x$-direction, connected in series with the head joint in the $y$-direction and connected in parallel with the bed joint in the $z$ direction. Eq.(14) represents the equilibrium at the cross-bed joint interface. It can be noted that the stress-strain state in the cross joint does not play a 
major role in the problem, because of its usually small volume ratio, so finer approximations are not considered.

Introducing Eqs.(13-14) in Eqs. (1-9) results in the elimination of all unknowns related to the cross joint. Further coupling with the nine elastic stress-strain relations in the unit, head joint and bed joint, namely,

$$
\begin{aligned}
& \varepsilon_{x x}^{k}=\frac{1}{E_{k}}\left[\sigma_{x x}^{k}-v_{k}\left(\sigma_{y y}^{k}+\sigma_{z z}^{k}\right)\right] \\
& \varepsilon_{y y}^{k}=\frac{1}{E_{k}}\left[\sigma_{y y}^{k}-v_{k}\left(\sigma_{x x}^{k}+\sigma_{z z}^{k}\right)\right], \quad \mathrm{k}=\mathrm{b}, 1,2, \\
& \varepsilon_{z z}^{k}=\frac{1}{E_{k}}\left[\sigma_{z z}^{k}-v_{k}\left(\sigma_{x x}^{k}+\sigma_{y y}^{k}\right)\right]
\end{aligned}
$$

yields a linear system of 18 equations. The unknowns are the six normal stresses and strains of the three components (unit, head joint and bed joint) and the shear stress and shear strain in the bed joint, amounting to a total of 20 unknowns.

Two additional equations are therefore needed to solve the problem. The equations can be derived introducing the shear deformation of the bed joint: the elastic mismatch between the normal $x$ strains in the unit and in the head joint is responsible for shear in the bed joint because of the staggered alignment of the units in a masonry wall. This mechanism is clear in Fig. 9 (where only the horizontal displacements have been magnified for sake of clearness) and leads to the approximated relation ${ }^{3}$ :

${ }^{3}$ If the assumed linear behaviour of $\varepsilon_{\mathrm{xx}}^{\mathrm{b}}$ in $\mathrm{x}$ is taken into account, it would lead to $\varepsilon_{\mathrm{xx}}^{\mathrm{b} 2}=\bar{\varepsilon}_{\mathrm{xx}}^{\mathrm{b}}+\sigma_{\mathrm{xy}}^{1} \frac{1-\mathrm{t}}{2 \mathrm{hE}_{\mathrm{b}}}$, but usually the second term in the right-hand side can be neglected. 


$$
\varepsilon_{x y}^{1}=\frac{1}{2} \times \frac{\Delta x_{2}-\Delta x_{b}}{2 t}=\frac{\varepsilon_{x x}^{2} \times t-\varepsilon_{x x}^{b 2} \times t}{4 t} \cong \frac{\varepsilon_{x x}^{2}-\bar{\varepsilon}_{x x}^{b}}{4}
$$

This relation holds in the hypothesis that the bed joint does not slip on the unit. With the additional elastic relation:

$$
\sigma_{x y}^{1}=2 G_{1} \varepsilon_{x y}^{1}
$$

a system of 20 equations and 20 variables is finally obtained. This linear system of equations can be solved numerically ${ }^{4}$ to give the internal stresses and strains for uniaxial load in the $i$-direction, given by

$$
\sigma_{i i}^{0}=1 \quad, \quad \sigma_{j j}^{0}=0 \quad(j \neq i) \quad, \quad i, j=x, y, z
$$

where $i$ represents the three orthogonal directions associated with the axis $x$, $y$ or $z$. The shear stress in the unit can be found by means of the internal equilibrium equation :

$$
\frac{\partial \sigma_{x x}^{b}}{\partial x}+\frac{\partial \sigma_{x y}^{b}}{\partial y}+\frac{\partial \sigma_{x z}^{b}}{\partial z}=0,
$$

which leads to :

$$
\sigma_{x y}^{b}=\sigma_{x y}^{1}\left(1-\frac{y}{h}\right)
$$

${ }^{4}$ It is noted that an explicit symbolic solution does exist and has been obtained. Nevertheless, the complexity of the solution precludes its use for practical purposes. The system of twenty equations can be easily reduced to a system of nine equations, which can be solved with any efficient linear solver. 
The homogenised Young's moduli and Poisson's coefficients of the basic cell are finally:

$$
E_{i}=\frac{\sigma_{i i}^{0}}{\varepsilon_{i i}} \quad, \quad v_{i j}=\left(\frac{\varepsilon_{j j}}{\varepsilon_{i i}}\right)_{i} \quad, \quad i, j=x, y, z
$$

where

$$
\begin{aligned}
& \varepsilon_{x x}=\varepsilon_{x x}^{1} \frac{l-t+2 t E_{1} / E_{3}}{l+t} \\
& \varepsilon_{y y}=\frac{\varepsilon_{y y}^{2}\left(h+2 t E_{2} / E_{3}\right)+h \varepsilon_{y y}^{b}}{2(t+h)} \\
& \varepsilon_{z z}=\varepsilon_{z z}^{b}
\end{aligned}
$$

and the subscript $i$ in the Young's modulus $E$ and the Poisson's ratio calculation ()$_{i}$ indicates that the values are calculated for uniaxial loading in the $i$-direction $(i=x, y, z)$.

\subsection{In-plane shear modulus $G_{x y}$}

The homogenised shear modulus $G_{x y}$ can be calculated by loading the basic cell with simple in-plane shear by means of suitable load and displacement fields. All external loads are zero on the basic cell boundary, except a uniform shear stress $\sigma_{x y}^{0}$ applied on the upper and lower face, and the equilibrium reactions $\sigma_{x y}$ on the left and right face. In this case the model neglects all stresses (and corresponding strains), except the in-plane shear in each basic cell component and the normal vertical component $\sigma_{y y}^{1}$ in the bed joint. Non-zero stress and strain components are assumed to be constant in each basic cell component, except $\sigma_{x y}$ in the unit, which 
must be a linear function of $\mathrm{x}$ to account for the effect of the normal stress $\sigma_{y y}^{1}$ in the bed joint. The deformation of the basic cell is approximated as shown in Fig. 10, with the bed joint in traction. Note that in the neighbouring basic cells (along $x$-axis) the bed joint is in compression, due to the antisymmetric loading conditions.

The internal stresses can be related by the equilibrium at adequately isolated parts of the composite:

$$
\begin{aligned}
& \text { (23) Interface brick - head joints } \quad \sigma_{x y}^{2}=\bar{\sigma}_{x y}^{b}+\frac{l}{2 h} \sigma_{y y}^{1} \\
& \text { Interface brick - bed joints } \quad \bar{\sigma}_{x y}^{b}=\sigma_{x y}^{1}
\end{aligned}
$$

where $\bar{\sigma}_{\mathrm{xy}}^{\mathrm{b}}$ is the mean value of $\sigma_{\mathrm{xy}}^{\mathrm{b}}$ in the unit.

The normal strain $\varepsilon_{\mathrm{yy}}^{1}$ can be derived from the geometric considerations in Fig. 10, where all the geometric quantities can be defined. Neglecting second order terms, it is straightforward to obtain:

$$
\varepsilon_{y y}^{1} \cong \frac{2 t^{\prime}-2 t}{2 t} \cong \frac{\Delta y+\frac{t}{l} \Delta y}{2 t} \quad, \quad \varepsilon_{x y}^{2}-\bar{\varepsilon}_{x y}^{b}=\frac{\Delta y}{2 t}+\frac{\Delta y}{2 l}
$$

which lead to :

$$
\varepsilon_{y y}^{1}=\varepsilon_{x y}^{2}-\bar{\varepsilon}_{x y}^{b}
$$

and, introducing the linear elastic relation between stress and strain, finally:

$$
\sigma_{y y}^{1}=E_{1}\left(\bar{\varepsilon}_{x y}^{b}-\varepsilon_{x y}^{2}\right)
$$

Eqs.(23,26), combined with the shear stress-strain relations

$$
\sigma_{x y}^{k}=2 G_{k} \varepsilon_{x y}^{k} \quad(\mathrm{k}=\mathrm{b}, 1,2)
$$


yield the shear stresses in the basic cell components:

(28)

$$
\begin{aligned}
\sigma_{x y}^{2} & =\sigma_{x y}^{0} \frac{l E_{1}+4 h G_{b}}{l E_{1}+4 h G_{b}+E_{1} \frac{l^{2}}{l+t}\left(\frac{G_{b}}{G_{2}}-1\right)}=k \sigma_{x y}^{0} \\
\sigma_{x y}^{1} & =\sigma_{x y}^{b}=\sigma_{x y}^{0} \frac{t+l}{l}-\frac{t}{l} \sigma_{x y}^{2} \\
\sigma_{y y}^{1} & =\left(\sigma_{x y}^{2}-\bar{\sigma}_{x y}^{b}\right) \frac{2 h}{l}
\end{aligned}
$$

The shear strains of the basic cell components and of the homogenised material, according to the deformation shown in Fig. 10, are related by the strain-displacement relations :

$$
\begin{array}{ll}
\varepsilon_{x y}^{1} \cong \frac{1}{2}\left(\frac{\Delta x_{1}}{2 t}-\frac{\Delta y}{l}\right), & \varepsilon_{x y}^{2} \cong \frac{1}{2}\left(\frac{\Delta x_{2}}{h}+\frac{\Delta y}{t}\right) \\
\bar{\varepsilon}_{x y}^{b} \cong \frac{1}{2}\left(\frac{\Delta x_{2}}{h}-\frac{\Delta y}{l}\right) \quad, & \varepsilon_{x y} \cong \frac{\left(\frac{\Delta x_{1}}{2}+\Delta x_{2}\right)}{2(h+t)}
\end{array}
$$

which lead to

$$
\varepsilon_{x y}=\frac{1}{h+t}\left[\left(l \bar{\varepsilon}_{x y}^{b}+t \varepsilon_{x y}^{2}\right) \frac{h}{l+t}+t \varepsilon_{x y}^{1}+\left(\varepsilon_{x y}^{2}-\bar{\varepsilon}_{x y}^{b}\right) \frac{t^{2}}{l+t}\right]
$$

The shear strains $\varepsilon_{x y}^{i}$ in the above equation can be calculated from the shear stresses given in Eq.(28) by means of the elastic relations of Eq.(27), resulting, finally, in the homogenised shear modulus $G_{x y}$

$$
G_{x y}=\frac{\sigma_{x y}^{0}}{2 \varepsilon_{x y}}=\frac{l(t+l)(t+h)}{k \frac{t l(t+h)}{G_{2}}+\frac{(t+l-k t)\left(l h-t^{2}\right)}{G_{b}}+\frac{t(t+l)(t+l-k t)}{G_{1}}}
$$

where $\mathrm{k}$ is defined in Eq.(28).

\subsection{Out-of-plane shear modulus $G_{x z}$}


To calculate the homogenised shear modulus $G_{x z}$, simple out-of-plane shear conditions in the $x z$-plane are imposed to the basic cell. Right and left faces are loaded with a uniform shear $\sigma_{\mathrm{xz}}^{0}$, while all other boundary stresses are zero, except the equilibrium reactions $\sigma_{x z}$ on front and rear face. Only out-of-plane shear stresses $\sigma_{x z}$ in each basic cell component and $\sigma_{y z}^{1}$ in the bed joint (and corresponding strains) are taken into account in the model, while all others are neglected. Non-zero stress and strain components are assumed to be constant, except $\sigma_{\mathrm{xz}}^{\mathrm{b}}$ which varies linearly in $x$ to account for the effect of $\sigma_{\mathrm{yz}}^{1}$ in the bed joint.

The deformation of the basic cell in this case is approximated as shown in Fig. 11, where one side has been fixed for the purpose of graphical clarity. The shear strain $\varepsilon_{\mathrm{yz}}^{1}$, with geometric considerations, can be found to be:

$$
\varepsilon_{y z}^{1}=\frac{1}{2} \frac{\Delta z}{2 t} \cong \frac{\Delta z_{2}-\frac{t}{l} \Delta z_{b}}{4 t}
$$

The following relations also hold:

$$
\begin{aligned}
& \text { Interface brick - head joint } \quad \sigma_{x z}^{2}=\bar{\sigma}_{x z}^{b}-\frac{(l-t)}{2 h} \sigma_{y z}^{1} \\
& \text { Right boundary } \quad h\left(\bar{\sigma}_{x z}^{b}+\frac{(l-t)}{2 h} \sigma_{y z}^{1}\right)+2 t \sigma_{x z}^{3}+h \sigma_{x z}^{2}=2(t+h) \sigma_{x z}^{0} \\
& \text { Interface cross - bed joints } \quad \sigma_{x z}^{3}=\sigma_{x z}^{1} \\
& \text { Interface brick - bed joint } \quad \Delta z_{b}=\Delta z_{1}
\end{aligned}
$$

By means of the shear stress-strain relations

$$
\sigma_{x z}^{k}=2 G_{k} \varepsilon_{x z}^{k} \quad(\mathrm{k}=\mathrm{b}, 1,2)
$$


and of the kinematic relations

$$
\varepsilon_{x z}^{1} \cong \frac{1}{2} \frac{\Delta z_{1}}{l} \quad, \quad \varepsilon_{x z}^{2} \cong \frac{1}{2} \frac{\Delta z_{2}}{t} \quad, \quad \bar{\varepsilon}_{x z}^{b} \cong \frac{1}{2} \frac{\Delta z_{b}}{l}
$$

Eqs.(32,33) yield :

$$
\begin{aligned}
& \varepsilon_{x z}^{1}=\varepsilon_{x z}^{b}=\sigma_{x z}^{0} \frac{t+h}{2\left(t G_{1}+h G_{b}\right)} \\
& \varepsilon_{x z}^{2}=\varepsilon_{x z}^{b} \frac{4 h G_{b}+(l-t) G_{1}}{4 h G_{2}+(l-t) G_{1}} \\
& \varepsilon_{y z}^{1}=\frac{1}{2}\left(\varepsilon_{x z}^{2}-\varepsilon_{x z}^{b}\right)
\end{aligned}
$$

and the homogenised shear modulus can be finally found as:

$$
G_{x z}=\frac{\sigma_{x z}^{0}}{2 \varepsilon_{x z}}=\frac{\sigma_{x z}^{0}}{2} \frac{t+1}{t \varepsilon_{x z}^{2}+l \varepsilon_{x z}^{b}}=\frac{(t+l)\left(t G_{1}+h G_{b}\right)}{(t+h)\left(t \frac{4 h G_{b}+(l-t) G_{1}}{4 h G_{2}+(l-t) G_{1}}+l\right)}
$$

\subsection{Out-of-plane shear modulus $G_{y z}$}

The basic cell in this case is assumed to be in simple out-of-plane shear (in the plane $y z$ ) by means of appropriate boundary conditions. The external load is a uniform shear stress $\sigma_{\mathrm{yz}}^{0}$ applied on upper and lower face of the basic cell, while equilibrium reactions $\sigma_{y z}$ act on front and rear face, where the boundary condition $\mathrm{u}_{\mathrm{y}}=0$ is imposed. Only the shear stresses $\sigma_{y z}$ (and corresponding strains) are taken into account in the model. It can be argued, from the deformation shown in Fig. 12 (where one side has been fixed for the purpose of graphical clarity), that

$$
\begin{array}{ll}
\text { Upper boundary } & t \sigma_{y z}^{2}+l \sigma_{y z}^{b}=(t+l) \sigma_{y z}^{0} \\
\text { Interface brick - bed joints } & \sigma_{y z}^{b}=\sigma_{y z}^{1} \\
\text { Interface brick - head joints } & \varepsilon_{y z}^{b}=\varepsilon_{y z}^{2}
\end{array}
$$


Combining these equations with the stress-strain relations

$$
\sigma_{y z}^{k}=2 G_{k} \varepsilon_{y z}^{k} \quad(\mathrm{k}=\mathrm{b}, 1,2)
$$

yields:

$$
\varepsilon_{y z}^{1}=\frac{G_{b}}{G_{1}} \varepsilon_{y z}^{b}=\frac{G_{b}}{G_{1}} \sigma_{y z}^{0} \frac{l+t}{2\left(l G_{b}+t G_{2}\right)}
$$

The homogenised strain is

$$
\varepsilon_{y z}=\frac{t \varepsilon_{y z}^{1}+h \varepsilon_{y z}^{b}}{t+h}
$$

and the homogenised shear modulus $\mathrm{G}_{\mathrm{yz}}$ is finally given as:

$$
G_{y z}=\frac{\sigma_{y z}^{0}}{2 \varepsilon_{y z}}=\frac{t+h}{t+l} G_{1} \frac{l G_{b}+t G_{2}}{t G_{b}+h G_{1}}
$$

\section{ELASTIC RESULTS}

The model described in the previous section has been applied to a real masonry basic cell and compared with the results of an accurate finite element analysis (FEA). This was considered a better evaluation of the analytical model that comparing analytical results with experimental results. In fact, the analytical model needs material data for the components and this type of data, at least for the mortar, always result from debatable assumptions or debatable interpretation of experimental results at the composite level (the curing conditions of mortar inside the composite are impossible to replicate, leading to meaningless results if the mortar specimens have been cured outside the composite). In the finite element 
analysis and the analytical model, the properties of the components can be taken absolutely equal.

The same elastic properties have been adopted for the bed joint, head joint and cross joint $\left(E_{1}=E_{2}=E_{3}=E_{m}, \quad v_{1}=v_{2}=v_{3}=v_{\mathrm{m}}\right)$. Different stiffness ratios between mortar and unit are considered. This allows to assess the performance of the model for inelastic behaviour. In fact, nonlinear behaviour is associated with (tangent) stiffness degradation and homogenisation of non-linear processes will result in large stiffness differences between the components. In the limit, the ratio between the stiffness of the different components is zero (or infinity), once a given components has no stiffness left. The unit dimensions are $210 \times 100 \times 52$ $\mathrm{mm}^{3}$ and the mortar thickness is $10 \mathrm{~mm}$. The material properties of the unit are kept constant, whereas the properties of the mortar are varied. For the unit, the Young's modulus $E_{b}$ is $20 \mathrm{GPa}$ and the Poisson's ratio $v_{b}$ is 0.15 . For the mortar, the Young's modulus is varied to yield a ratio $E_{b} / E_{m}$ ranging from 1 to 1000 . The Poisson's ratio $v_{m}$ is kept constant to 0.15 .

The adopted range of $E_{b} / E_{m}$ is very large (up to 1000), if only linear elastic behaviour of mortar is considered. However, those high values are indeed encountered if inelastic behaviour is included. In such case, $E_{b}$ and $E_{m}$ should be understood as linearised tangent Young's moduli, representing a measure of the degradation of the (tangent / secant) stiffness matrices utilised in the numerical procedures adopted to solve the non-linear problem. Note that the ratio $E_{b} / E_{m}$ tends to infinity when softening of the mortar is complete and only the unit remains structurally active. 
The elastic properties of the homogenised material, calculated by means of the proposed micro-mechanical model, are compared in Fig. 13 with the values obtained by FE analysis. The agreement is very good in the entire range $1 \leq E_{b} / E_{m} \leq 1000$. Fig. 14 gives the relative error of the elastic parameters predicted by the proposed model and show that it is always $\leq$ 6\%. The thinner curves in Fig. 14 ("simplified model") give the results of a simplified model ( $E_{x}$ only), derived from the model presented in the paper, but where the deformation mechanisms of the bed joint, mentioned in Sec.3.1, have not been taken into account. The simplified model therefore neglects the main effects due to the misalignment of the units in the masonry wall and coincides with the full model when the units are aligned in the wall. The simplified model is, therefore, closer to the standard twostep homogenisation referred to in Chapter 1. Fig. 14 also includes the results of the standard two-step homogenisation of Lourenço (1997). A nonacceptable error up to $45 \%$ is found in such case, for the homogenisation of the elastic Young's modulus along the $x$ direction. Directions $y$ and $\mathrm{z}$ are not shown in the picture for the sake of clarity of the picture. Less pronounced differences are found in these directions as the unit geometry if oriented in the $x$ direction and the running bond reduces largely the influence of the head joint for homogenisation in the y direction, see Lourenço (1997).

For large ratios $E_{b} / E_{m}$ the simplified model predicts value of $E_{x}, v_{x z}$ and $G_{x z}$ much smaller than the actual values obtained by FE analysis. The large and increasing errors of the simplified model on these variables (up to 50\%) indicate that for very degraded mortar the neglected deformation mechanisms of the bed joint contribute significantly to the overall basic cell 
behaviour. In the proposed micro-mechanical model the in-plane shear resistance of the bed joint $\left(\sigma_{x y}^{1}\right)$ is responsible for the increased stiffness in the $x$-direction (up to $50 \%$ ), which could not be accounted for only with the normal stresses in the unit and in the mortar. This increase of the stiffness in $x$ yields also higher Poisson's coefficient in $y$ and $z$. The vertical normal stress in the bed joint $\left(\sigma_{y y}^{1}\right)$ contributes to the in-plane shear stiffness, while the out-of-plane shear $\left(\sigma_{y z}^{1}\right)$ can double (for very large ratios $E_{b} / E_{m}$ ) the shear resistance of the basic cell to a shear load $\sigma_{x z}^{0}$ calculated with the simplified model.

\section{A HOMOGENISED FAILURE CRITERION}

Failure of quasi-brittle materials such as concrete and masonry is a difficult issue. Even for apparently simple loading conditions such as uniaxial compression, failure mechanisms denoted as Mode I, Mode II or local crushing are the object of a long-going debate among researchers, see van Mier (1998) for a discussion. For masonry under uniaxial compression, a lot of researchers claim that mortar is subjected to triaxial compression and the unit is in a mixed uniaxial compression - biaxial tension, see e.g. Hendry (1998). The assumption that failure of masonry is governed solely by the tensile failure of the unit, induced by the expansion effect of mortar, is certainly highly debatable because the influence of the micro-structure 
(voids, inclusions, etc.) is also a key issue. A discussion on these aspects is out of the scope of the present paper and will not be carried out.

The sole objective of this section is to demonstrate that the shape of the anisotropic failure surface based on the micro-mechanical homogenised model is reasonable and seems to be able to reproduce experimental results available in the literature. A direct connection to the triggered failure modes is not the issue here. Currently, a research project being carried out at University of Minho is addressing these issues.

The homogenised micro-mechanical model allows to calculate not only the homogenised material properties of the basic cell, but also stresses and strains in each basic cell component. Making use of the superposition principle, holding up to failure if an elastic-brittle behaviour is assumed for mortar and unit, the stress distribution for an arbitrary loading case can be derived by linear combination of the solutions of the six basic problems presented in Chapter 3.

Then, the failure load for the homogenised cell results from reaching the failure criteria of any of the two components. For the purpose of this section, the simplest failure criteria can be considered for the unit and mortar. Assuming that both materials are isotropic, the Rankine yield surface has been assumed to describe the tensile behaviour, while the classic von Mises criteria has been adopted to describe the compression behaviour, see Fig.14. These are defined by

Rankine: $\quad \sigma_{p}^{k}=\sigma_{t}^{k}$ 


$$
\text { Von Mises } \quad: \quad \sigma_{\text {Mises }}^{k}=\sigma_{c}^{k} \quad ; \quad \mathrm{k}=1,2, \mathrm{~b}
$$

where $\sigma_{p}^{k}$ is the maximum principal tensile stress, $\sigma_{\text {Mises }}^{k}$ is the equivalent von Mises stress, and $\sigma_{t}^{k}, \sigma_{c}^{k}$ are the tensile and compressive strengths of the component $k$. It is stressed that von Mises is hardly acceptable as a failure criterion for frictional materials subjected to general threedimensional stress states, which is not the case here. On the contrary, it can approximate failure in the compression-compression regime or the tensioncompression regime for plane stress problems, as adopted here. It has been used for this purpose by a number of authors.

Fig. 16 shows the resulting failure surfaces in the plane stress space $\left(\sigma_{1}, \sigma_{2}\right)$ for a test case, where the principal loading stress directions coincide with the material axes, i.e. only in-plane normal stresses $\sigma_{1}, \sigma_{2}$ and no shearing are applied on the cell faces. The material and geometric parameters for unit and mortar, which are defined in the picture, aim at reproducing the results from Page $(1981,1983)$. In the micro-mechanical model, the principal directions in the bed joint do not coincide with the material axes even in the absence of shear loading, due to presence of shear in the bed joint. The intersection of all failure surfaces (the thicker line in Fig. 16a which is reproduced in Fig. 16b) is the failure surface of the homogenized basic cell. In the unit, due to the variation of $\sigma_{x x}^{b}$ with $\mathrm{x}$, the compression failure criteria is applied to the point which leads to a maximum of the von Mises stress, which can be easily calculated. 
The stresses in Fig. 16 have been normalised by the mortar tensile strength $\left(\sigma_{t m}\right)$ for the purpose of comparison with experimental results. It can be noted that the plot of the yield stress in the unit of Fig. 16a is not a perfect ellipse (check top and bottom parts): actually it is the intersection (worst value) of two different von Mises ellipses, corresponding to the maximum and minimum values of the stress $\sigma_{x x}^{b}$, which has been assumed to vary linearly with $x$ in the unit. For a given stress path, the failure loads and the type of failure mechanism depend strictly on geometry, on elastic material properties and above all on the relative material strengths of the different cell components. Note that the direction of the maximum principal stress in each component does not correspond always to the same material direction, but does change with the load ratio $\sigma_{1} / \sigma_{2}$. Additionally, the tensile stress of the unit in the compression-compression range is $\sigma_{z z}$ as the lateral expansion in $z$ of the mortar (prevented in $x$ and $y$ by the biaxial compression) is the cause of a tensile stress state of the unit in the direction $Z$.

According to the proposed model, Fig. 16b shows that, for the selected material and geometric properties, failure by tension of the head joint is expected in the tension-compression range, while tension in the bed joint is the cause of the failure in the compression-tension range. In the compression-compression range, three mechanisms are responsible of the failure of the cell for decreasing $\sigma_{1} / \sigma_{2}$ ratios: tensile failure in the bed joint (for very high ratios), compressive failure in the head joint and compressive failure in the bed joint. Again, it is believed that these conclusions are 
debatable and more research is needed on the issue of compressive failure of masonry.

Nevertheless, a comparison between the results obtained with the micromechanical model and the experimental results of Page $(1981,1983)$ are given in Fig. 17. The agreement in the actual values is misleading as the parameters of the micromechanical model were fitted to obtain the actual uniaxial strengths exhibited in the experiments. Nevertheless, very good agreement is found in the shape of the yield surface, indicating that the proposed model can be used as a possible macro-model to represent the composite failure of masonry. Such an approach might reduce the effort to develop and implement specific complex macro-models for the composite behaviour of masonry such as in Lourenço et al. (1998).

It is stressed that the present work is, at this stage, mostly fundamental and represents a contribution to researchers working in the homogenisation field. Homogenisation methods represent powerful tools available for analysts, but are not yet fully developed. The aim of this section is only to demonstrate that an anisotropic failure criterion similar to the criteria observed experimentally can be obtained. Given the difficulties in adequately measuring mortar and interface properties, i.e. the absence of adequate experimental values to assess the model, and the actual simplicity of the model, the analytical results presented seem of value to the authors.

Finally, it must be stressed that failure by tension of the head joints will not imply necessarily the failure of the composite system in the macroscale, as adopted in this paper. For the simplified approach used here, this seems the most reasonable assumption (i.e. if the weakest link fails, the system 
fails). The issue of actual non-linear behaviour of the components with progressive stiffness degradation must be assessed elsewhere. The definition of failure is a tricky issue for a composite material such masonry. The wellknow experimental results of Page $(1981,1983)$ indeed result from a definition of failure in compression as early splitting of the bed joints in tension, in the case of compression parallel to the bed joints, see Dhanasekar et al. (1985).

\section{CONCLUSIONS}

This paper presents a novel micro-mechanical homogenisation model for masonry, which includes additional deformation modes of the basic cell. From a comparison with the results obtained in a detailed finite element simulation of the basic cell, it is demonstrated that relatively small errors occur in the homogenisation process, by including these mechanisms. The proposed one-step homogenisation represents a major development with respect to the standard two-step homogenisation process, head and bed joints being introduced successively, in which very large errors occur for large differences between the unit and mortar stiffness, Lourenço et al. (1998).

Finally, it is shown that the anisotropic failure surface obtained from the proposed micro-mechanical model, assuming elastic-brittle behaviour of unit and mortar, seems to, qualitatively, reproduce well the experimental results available for the composite behaviour of masonry. The quantitative 
assessment of the model cannot be addressed at this stage, due to the reduced experimental data available. It is expected that interface behaviour and progressive stiffness degradation must be included in the simplified homogenisation techniques to assess the their quantitative performance.

\section{Acknowledgements}

A. Zucchini was partially supported by GIANO project funded by EU and MURST.

P.B. Lourenço was partially supported by project PRAXIS-C-ECM13247-1998 funded by the Portuguese Science and Technology Foundation (FCT).

\section{REFERENCES}

Anthoine, A., 1995. Derivation of the in-plane elastic characteristics of masonry through homogenization theory, International Journal of Solids and Structures 32(2), 137-163.

Anthoine, A., 1997. Homogenisation of periodic masonry: Plane stress, generalised plane strain or 3D modelling?, Communications in Numerical Methods in Engineering 13, 319-326.

Bakhvalov, N., Panasenko, G., 1989. Homogenization: Averaging processes in periodic media. Kluwer Academic Publishers, Dordrecht, The Netherlands. 
Bati, S.B., Ranocchiai, G., Rovero, L., 1999. A micromechanical model for linear homogenization of unit masonry, Materials and Structures 32, 22-30.

Besdo, D., 1985. Inelastic behaviour of plane frictionless block systems described as Cosserat media, Archives in Mechanics 37(6), 603619.

Dhanasekar, M., Page, A.W. and Kleeman, P.W., 1985. The failure of brick masonry under biaxial stresses, Proc. Intsn. Civ. Engrs., Part 2, 79, 295-313.

Hendry, A.W., 1998. Structural Masonry. Macmillan Press, United Kingdom.

Lofti, H.R., Shing, P.B., 1994. Interface model applied to fracture of masonry structures, Journal of Structural Engineering ASCE 120(1), 63-80.

Lopez, J., Oller, S., Oñate, E., Lubliner, J., 1999. A homogeneous constitutive model for masonry, International Journal for Numerical Methods in Engineering 46, 1651-1671.

Lourenço, P.B., 1996. A matrix formulation for the elastoplastic homogenisation of layered materials, Mechanics of Cohesive-Frictional Materials 1, 273-294.

Lourenço, P.B., 1997. On the use of homogenisation techniques for the analysis of masonry structures, Masonry International 11(1), 26-32.

Lourenço, P.B., Rots, J.G., 1997. A multi-surface interface model for the analysis of masonry structures, Journal of Engineering Mechanics ASCE 123(7), 660-668. 
Lourenço, P.B., Rots, J.G., Blaauwendraad, J., 1998. Continuum model for masonry: Parameter estimation and validation, Journal of Structural Engineering ASCE 124(6), 642-652.

Maier, G., Papa, E., Nappi, A., 1991. On damage and failure of unit masonry. In: Experimental and numerical methods in earthquake engineering. Balkema, Brussels and Luxenbourg, pp. 223-245.

van Mier, J.G.M., 1998. Failure of concrete under uniaxial compression: An overview. In: Fracture mechanics of concrete structures, Vol. 2. Aedificatio, Freiburg, pp. 1169-1182.

Mühlhaus, H.-B., 1993. Continuum models for layered soil and blocky rock. In: Comprehensive rock engineering, Vol. 2. Pergamon Press.

Page, A.W., 1981. The biaxial compressive strength of brick masonry, Proceedings from the Institution of Civil Engineers - Part 2 - 71, 893-906.

Page, A.W., 1983. The strength of brick masonry under biaxial compression-tension, International Journal of Masonry Construction 3(1), 26-31.

Pande, G.N., Liang, J.X., Middleton, J, 1989. Equivalent elastic moduli for unit masonry, Computers and Geotechnics 8, 243-265.

Pietruszczak, S., Niu, X., 1992. A mathematical description of macroscopic behavior of unit masonry, International Journal of Solids and Structures 29(5), 531-546.

van der Pluijm, R., 1999. Out of plane bending of masonry: Behaviour and strength. Ph.D. Dissertation, Eindhoven University of Technology, The Netherlands. 
Urbanski, A., Szarlinski, J., Kordecki, Z., 1995. Finite element modeling of the behavior of the masonry walls and columns by homogenization approach. In: Computer methods in structural masonry - 3, Books \& Journals International, Swansea, pp. 32-41. 

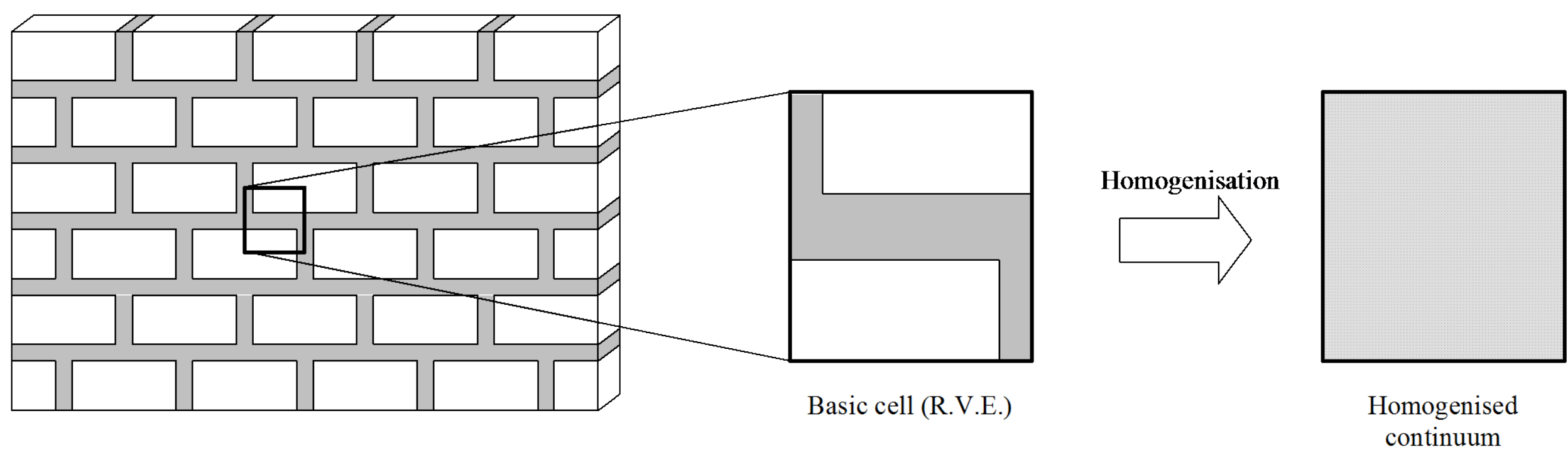

Fig. 1 - Basic cell for masonry and objective of homogenisation 


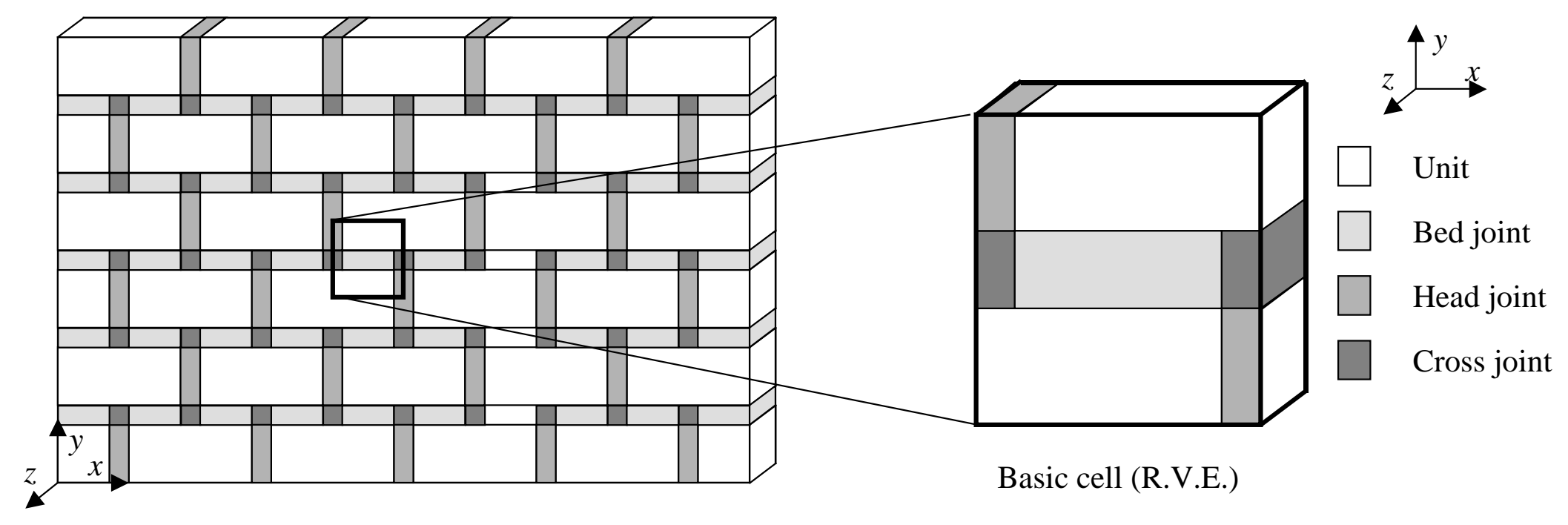

Fig. 2 - Definition of (a) masonry axes and (b) masonry components considered in the analysis: unit, head joint, bed joint and cross joint 


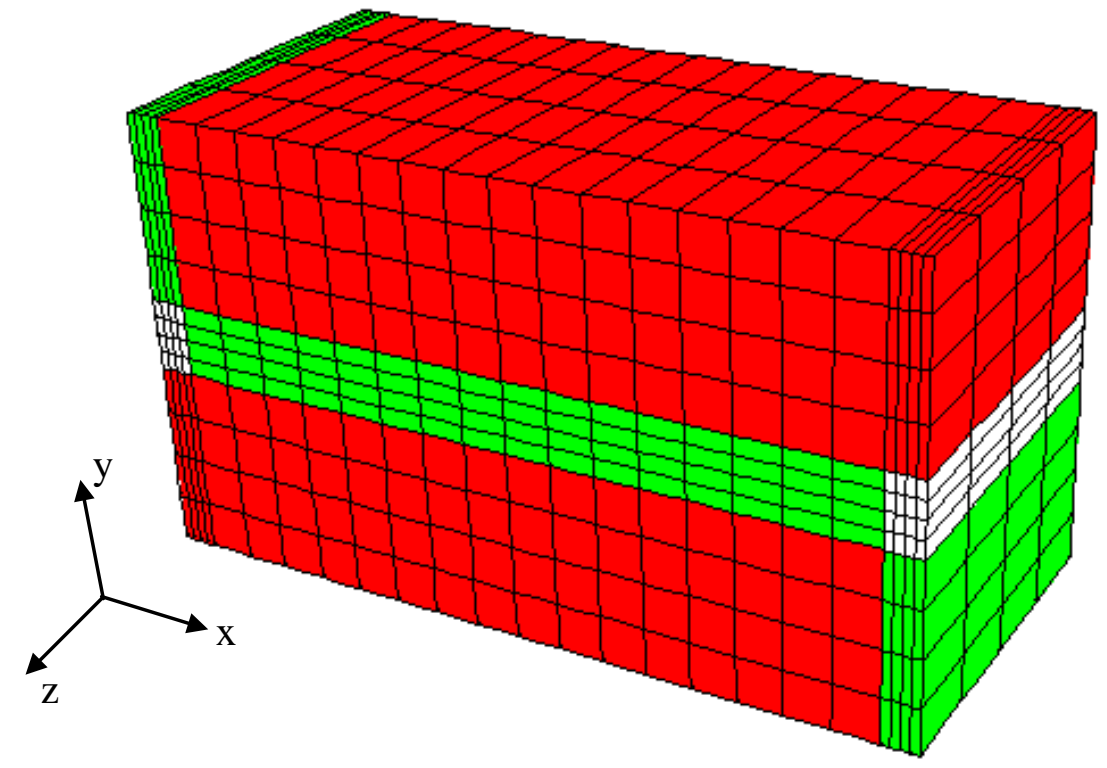

Fig. 3 - Finite element mesh for the basic cell adopted in the analyses 


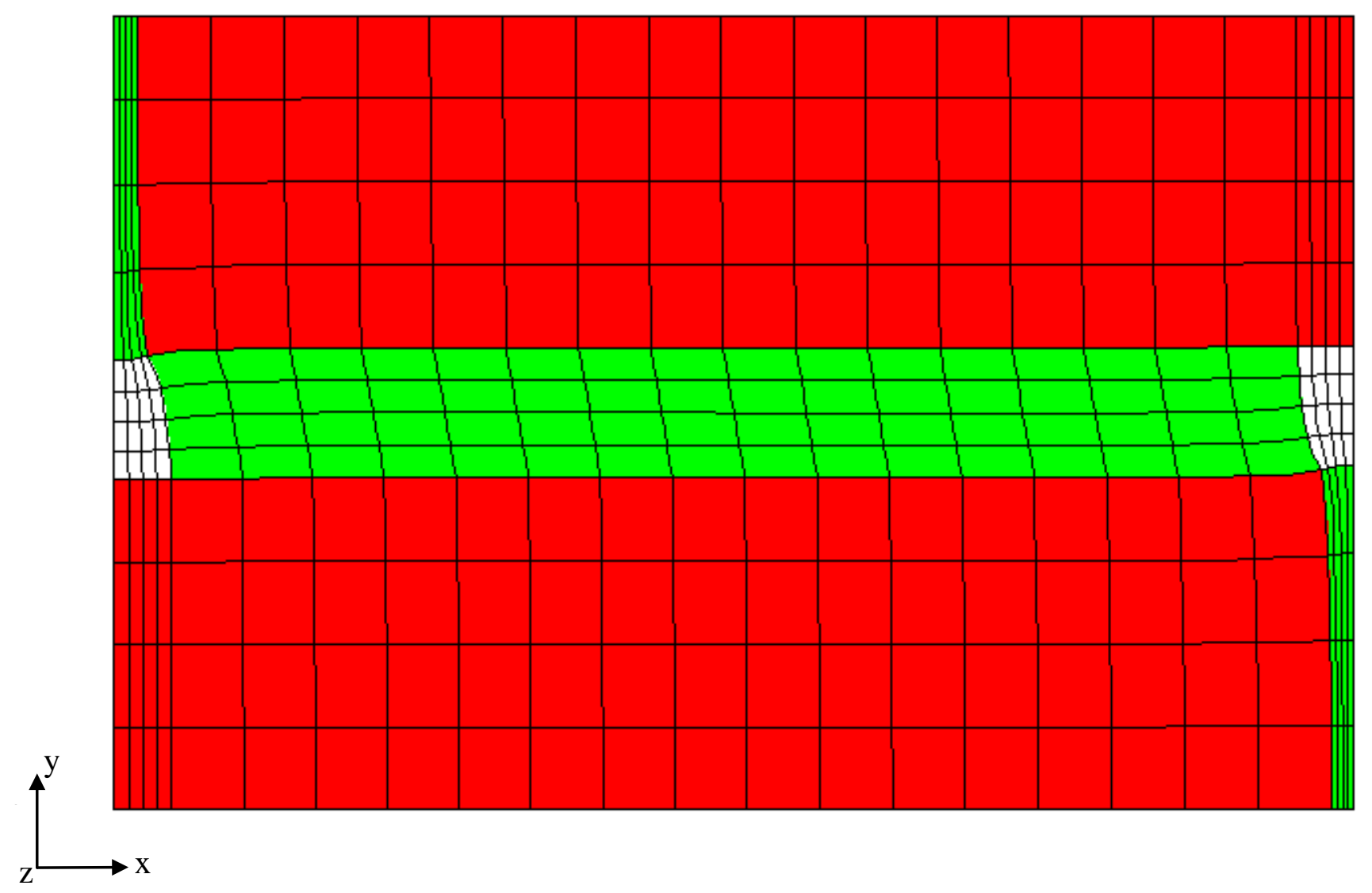

(a) X direction 


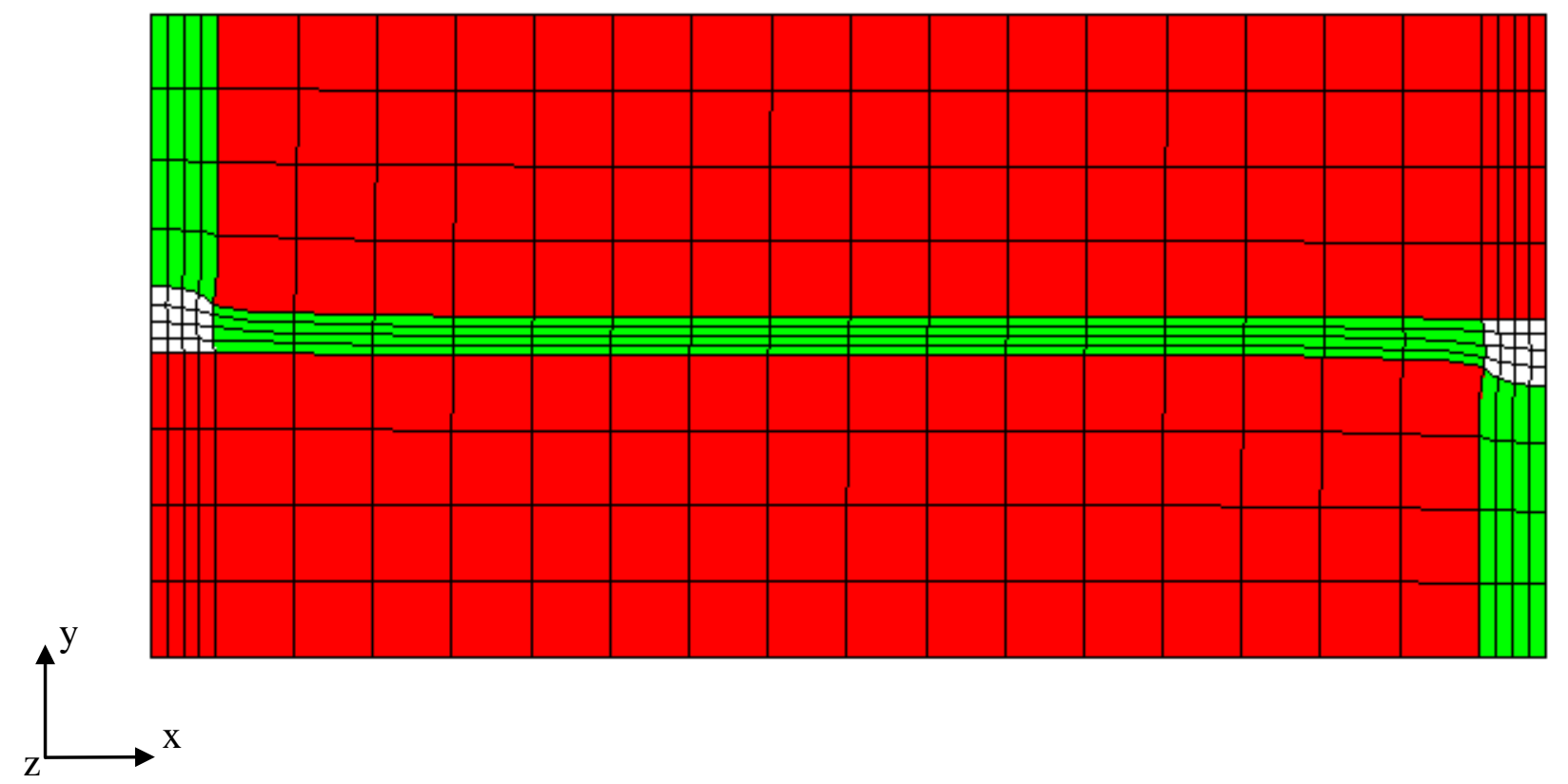

(b) Y Direction 


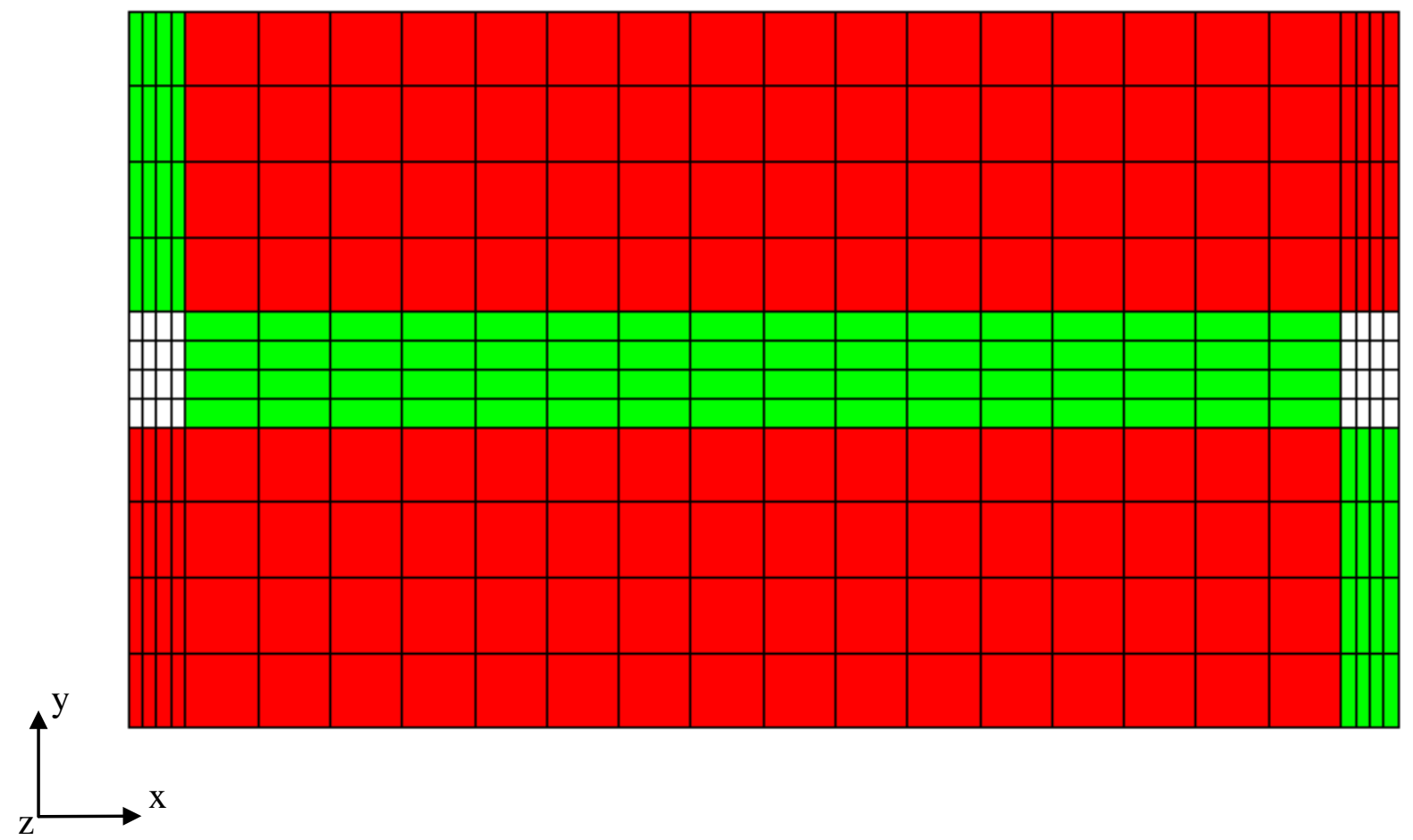

(c) Z Direction 


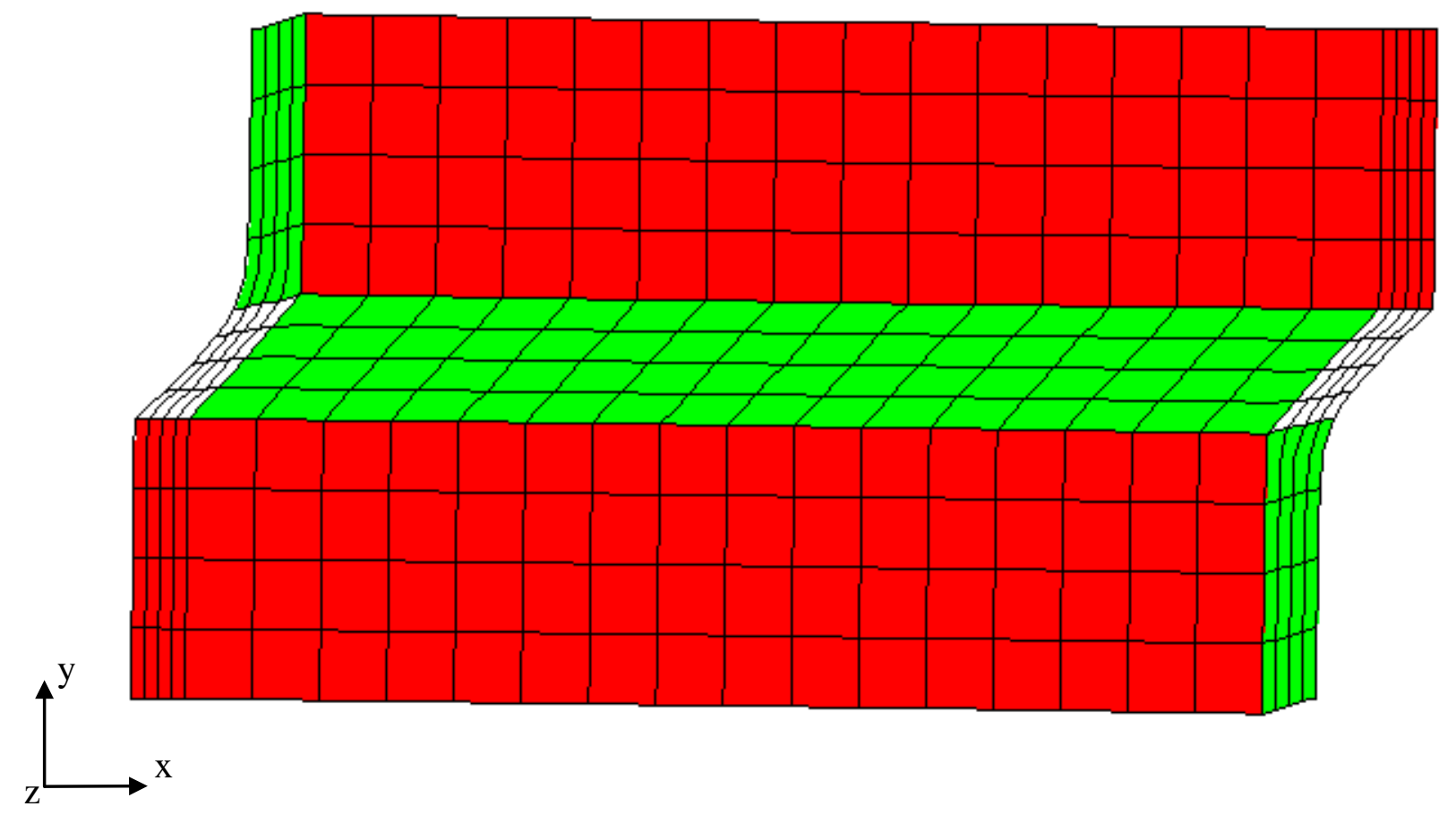

(d) XY Direction 


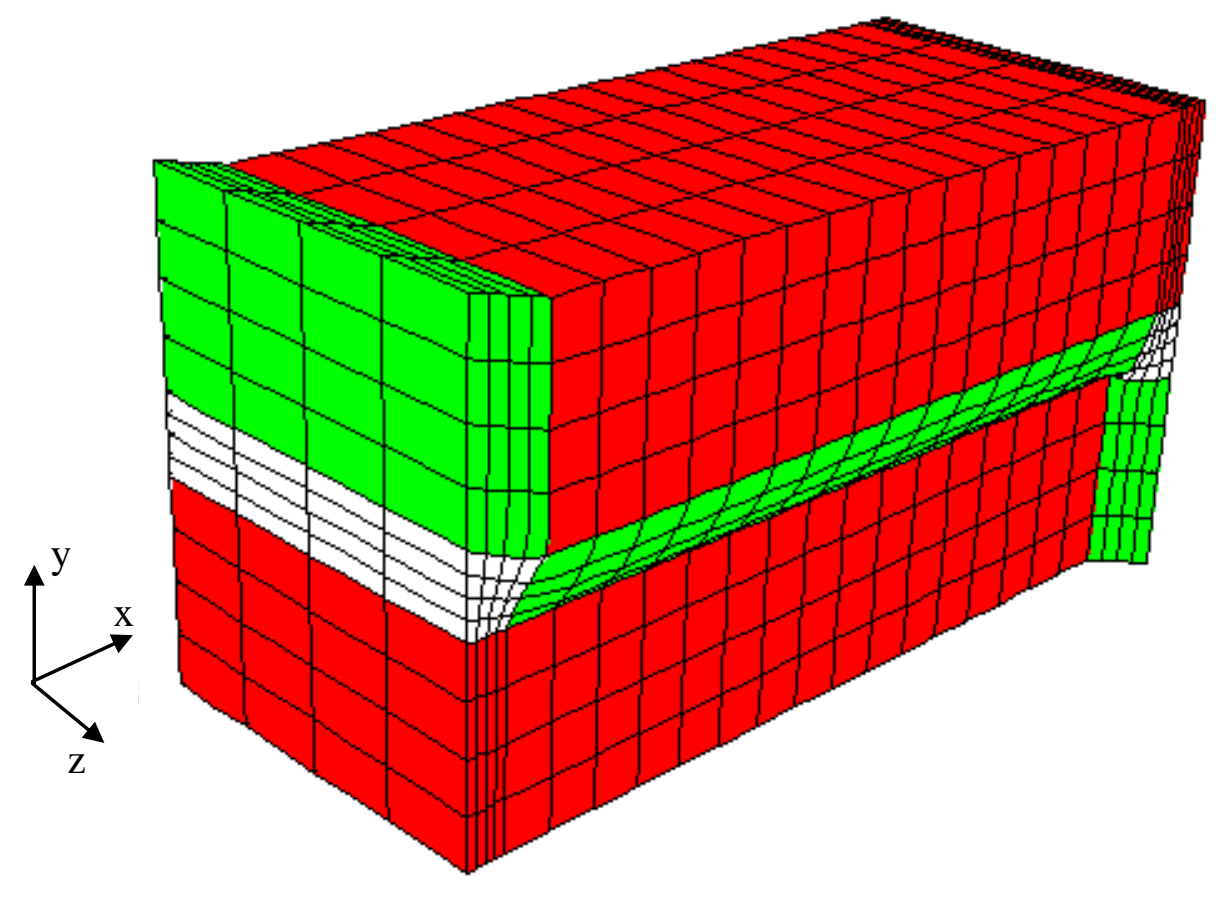

(e) XZ Direction 


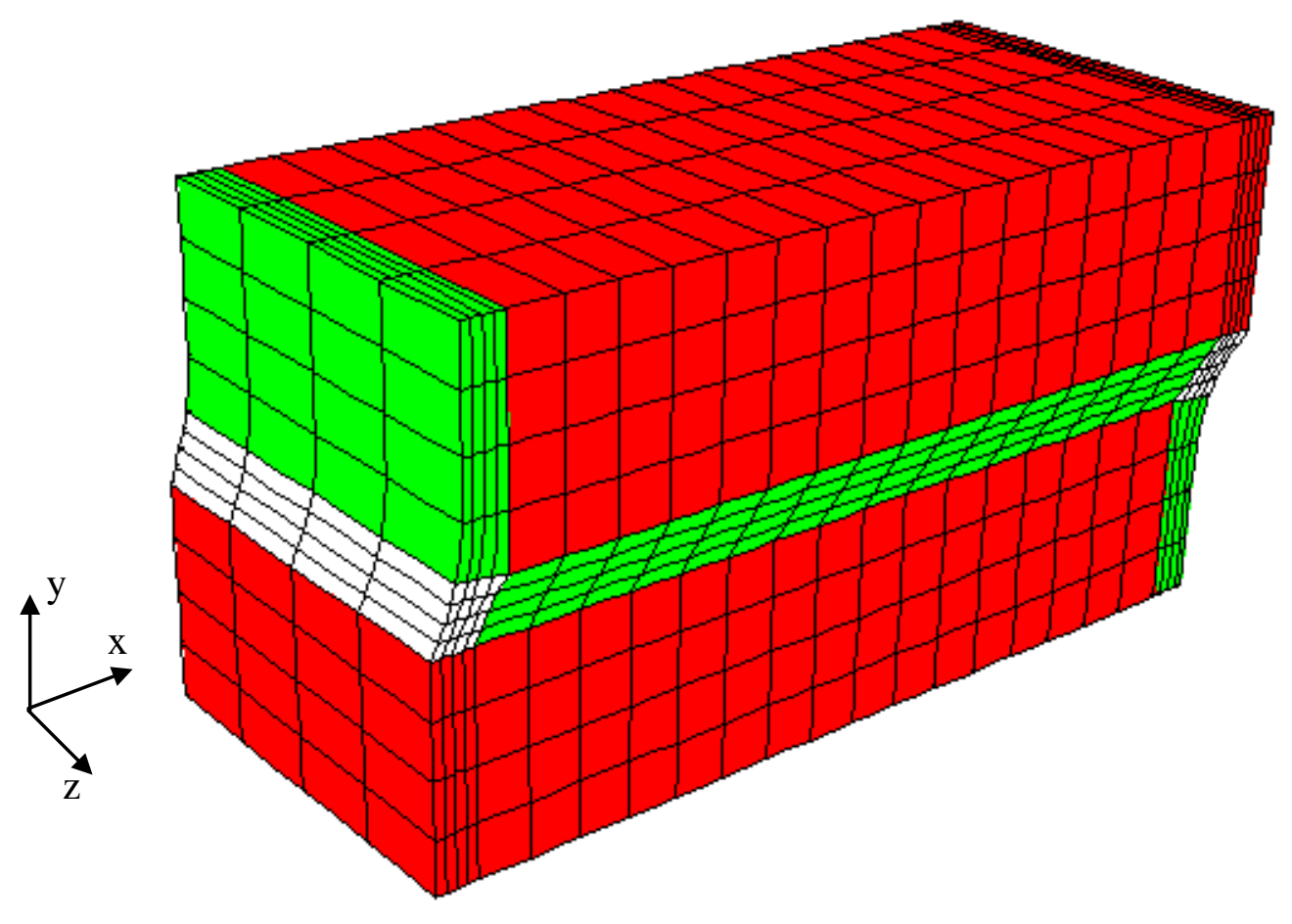

(f) YZ Direction

Fig. 4 - Deformed configuration resulting from the finite element analysis on the basic cell: (a) compression $x$, (b) compression $y$, (c) compression $z$, (d) shear $x y$, (e) shear $x z$ and (f) shear $y z$. 


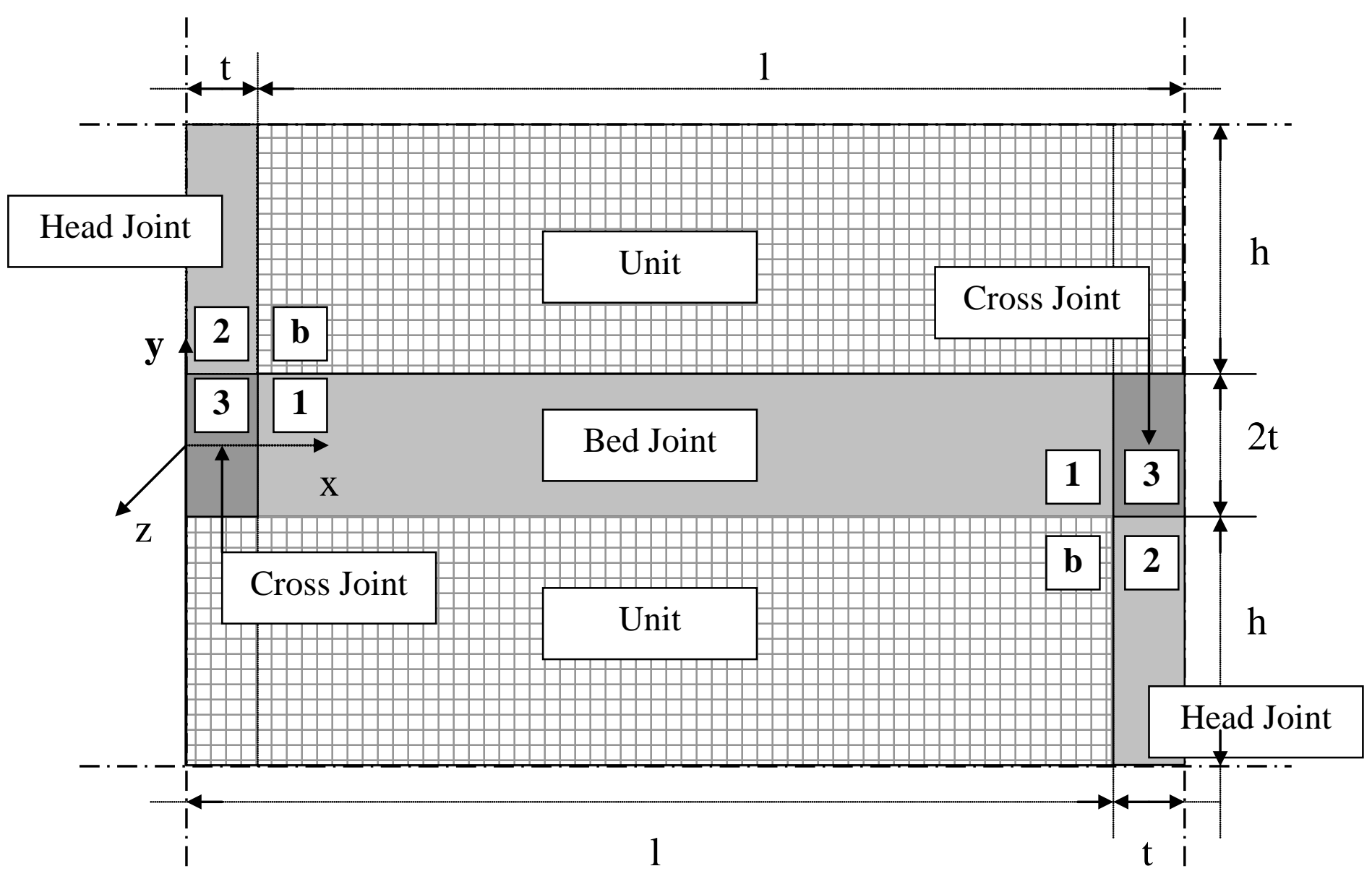

Fig. 5 - Adopted geometry symbols. 


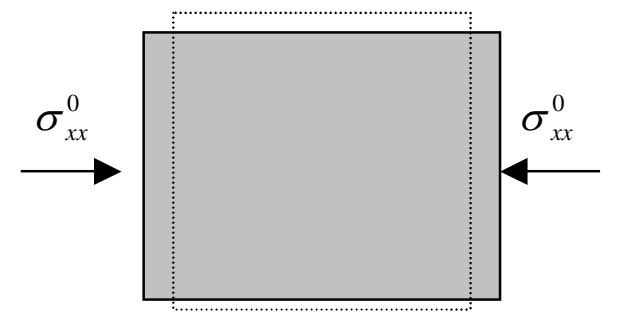

(a)
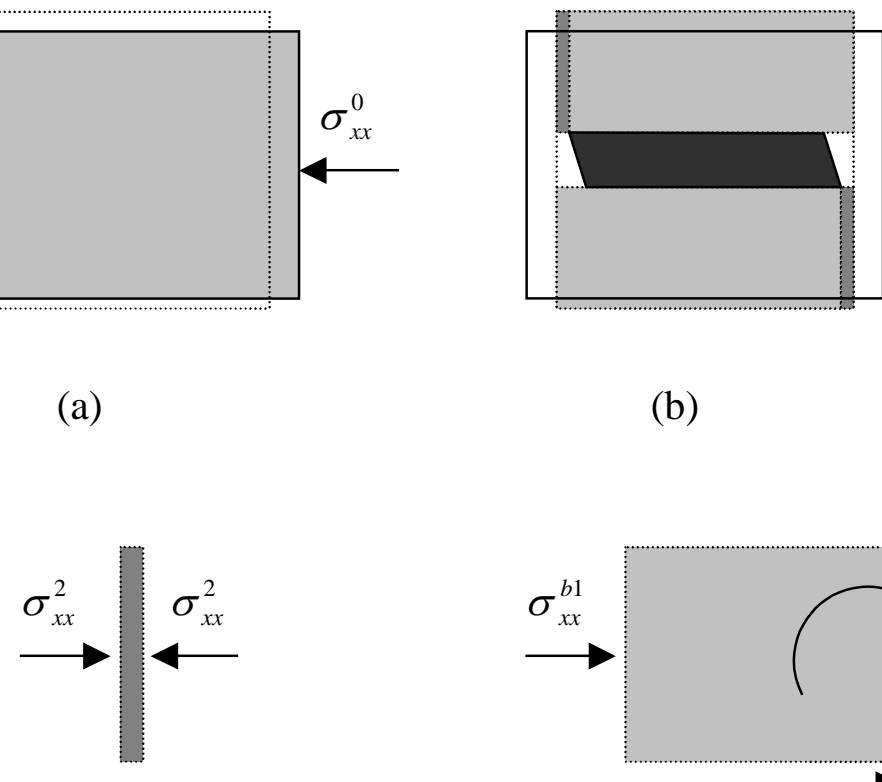

$\square$ Cross joint (3)

$\square$ Unit (b)

$\square$ Head joint (2)

Bed joint (1)

(b)

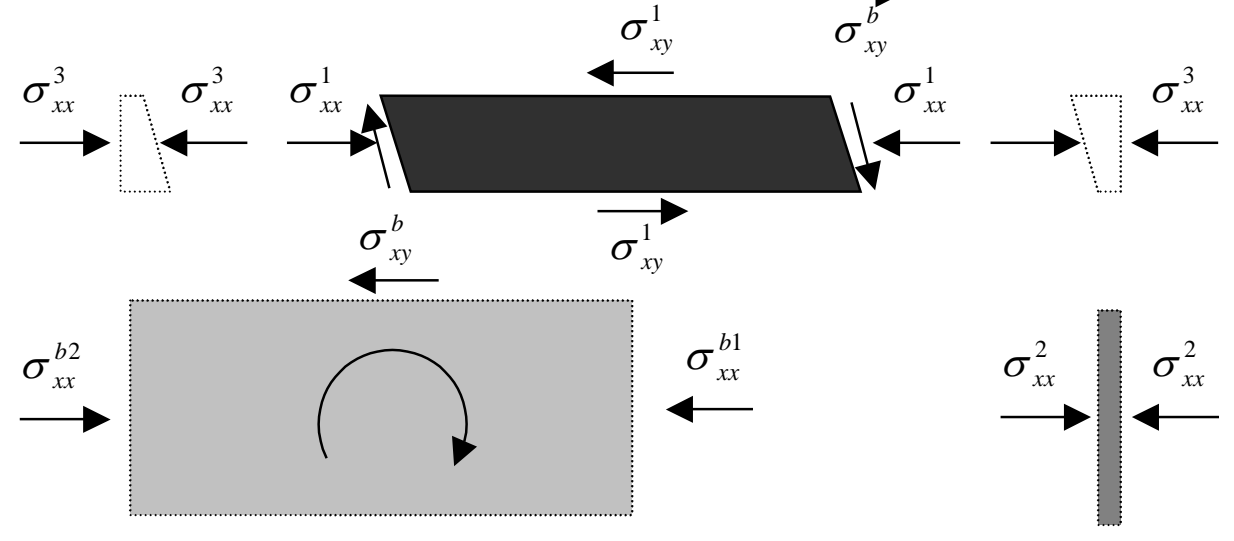

(c)

Fig. 6 - Normal stress loading parallel to the $x$ axis: (a) equivalent homogenised cell; (b) assumed deformation behaviour; (c) assumed involved stress components 


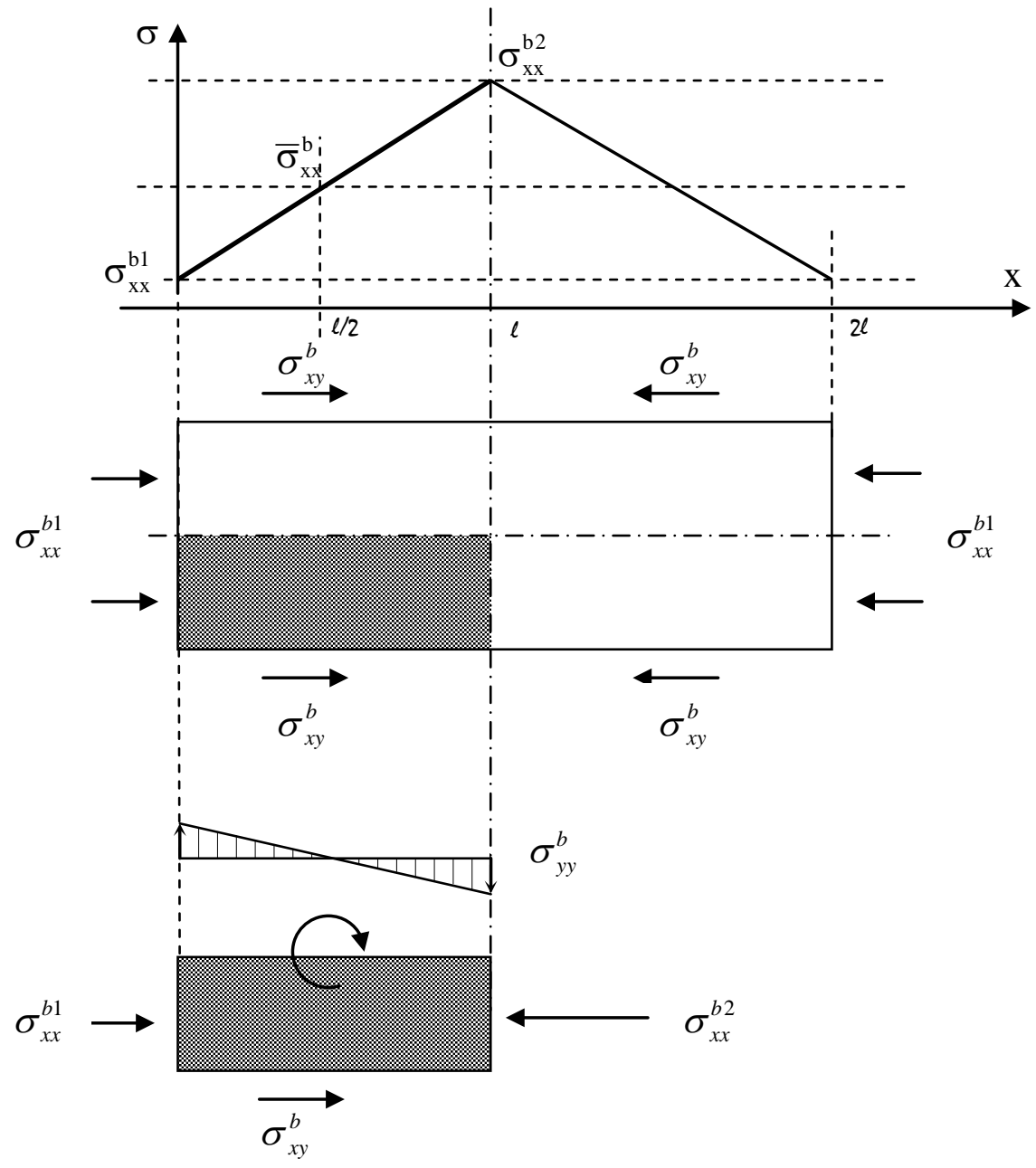

Fig. 7 - Normal stress loading parallel to the $x$ axis: unit equilibrium (couple moment equal to self-equilibrating vertical stress distribution) 45 


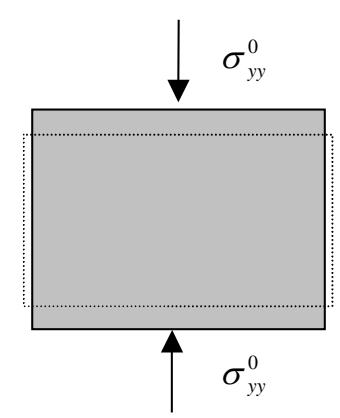

(a)

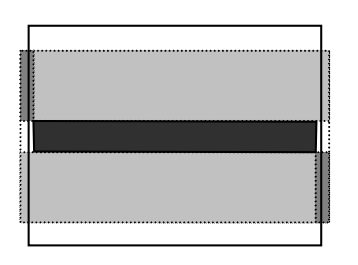

(b)

\section{$\square$ Cross joint (3)}

$\square$ Unit (b)

$\square$ Head joint (2)

— Bed joint (1)

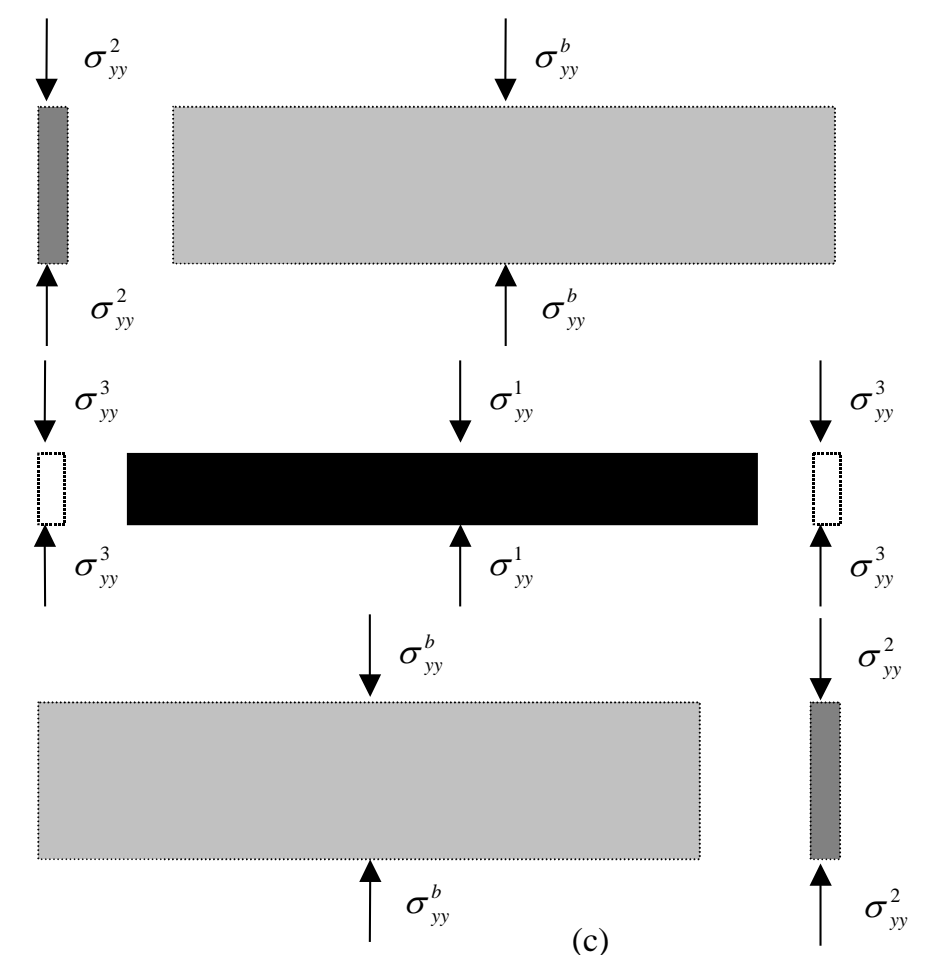

(c)

Fig. 8 - Normal stress loading parallel to the $y$ axis: (a) equivalent homogenised cell; (b) assumed deformation behaviour; (c) assumed involved stress components 


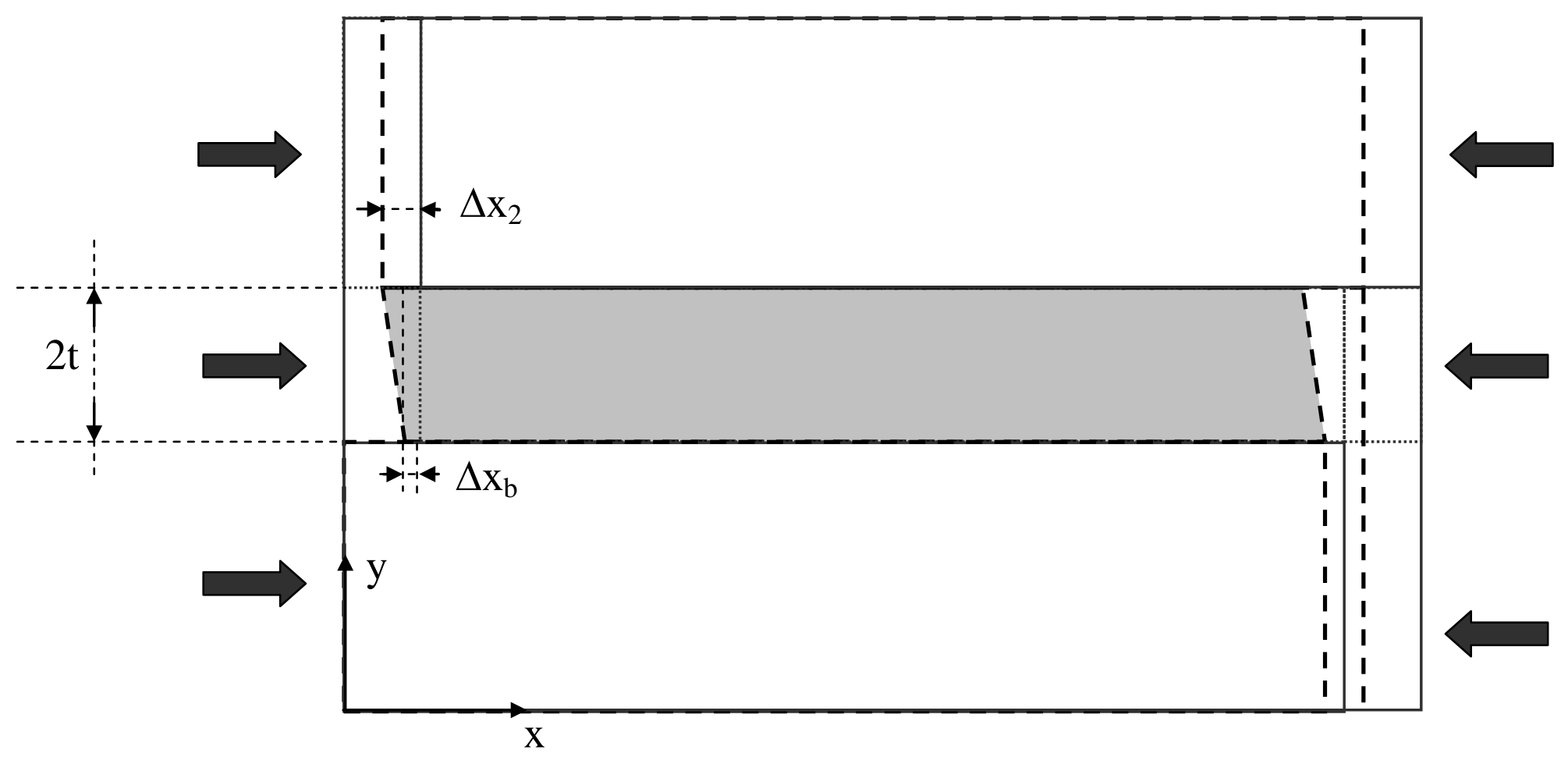

Fig. 9 - Model assumptions for compression along the $x$ axis 


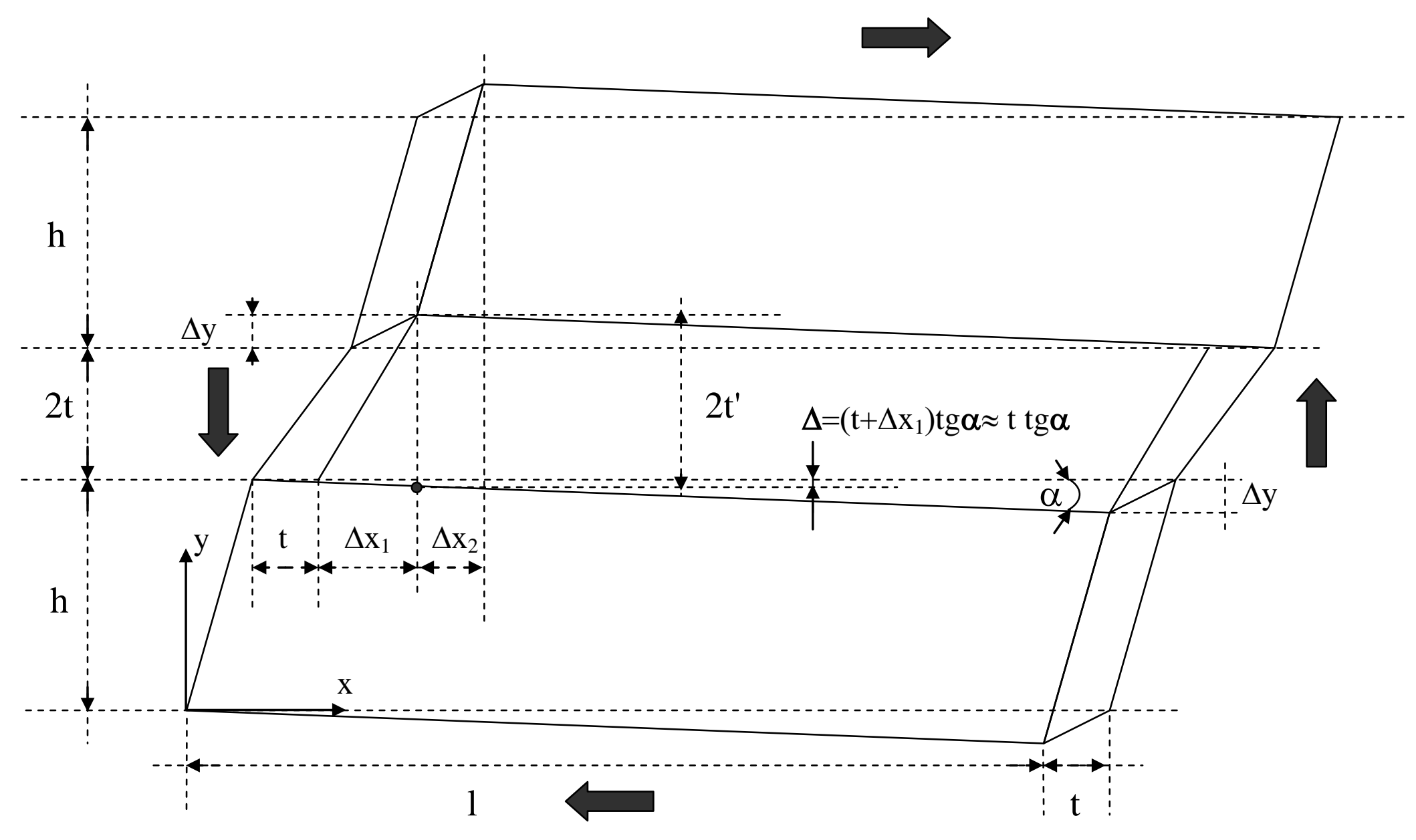

Fig. 10 - Model assumptions for $x y$ shear 


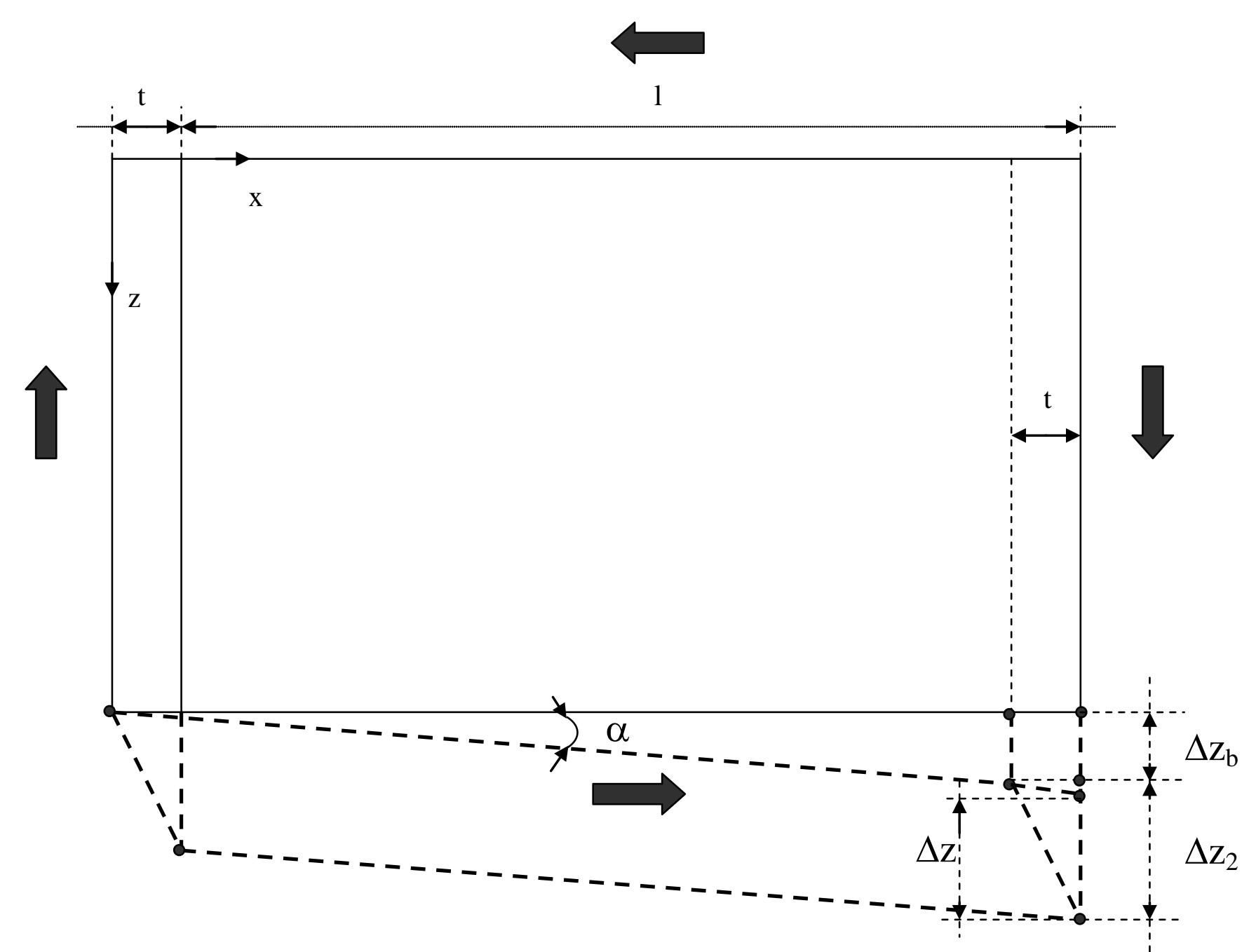

Fig. 11 - Model assumptions for $x z$ shear 


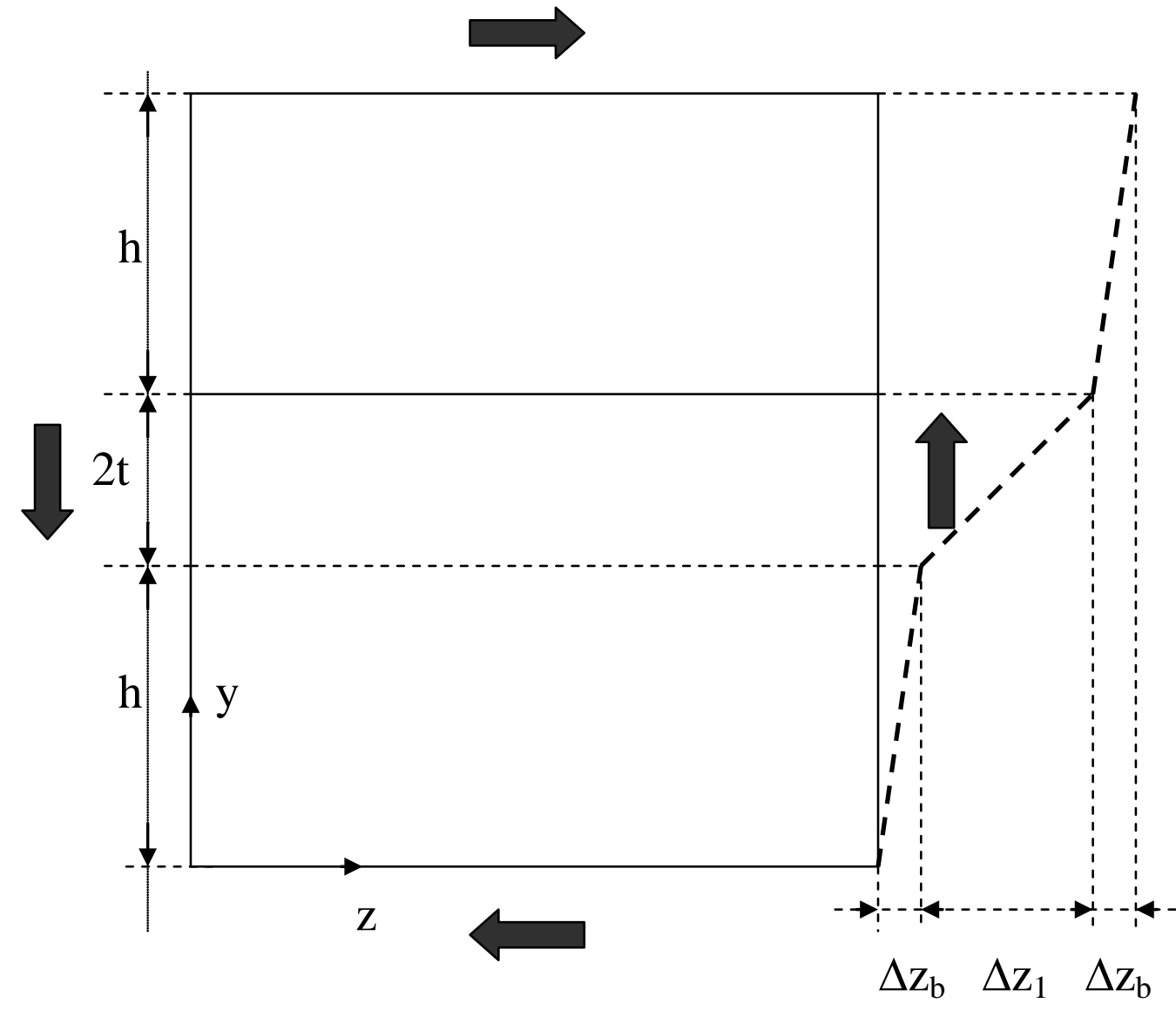

Fig. 12 - Model assumptions for $y z$ shear 
Titolo:

/disk6/zucchini/OFFICE/PLOT/EmortE1.eps

Autore:

xmgr

Anteprima:

L'immagine EPS non è stata salvata

con l'anteprima inclusa in essa.

Commento:

L'immagine EPS potrà essere stampata con una stampante

PostScript e non con

altri tipi di stampante.

(a) 


\section{Titolo:}

/disk6/zucchini/OFFICE/PLOT/EmortNU1.eps

Autore:

xmgr

Anteprima:

L'immagine EPS non è stata salvata

con l'anteprima inclusa in essa.

Commento:

L'immagine EPS potrà essere stampata con una stampante

PostScript e non con

altri tipi di stampante. 


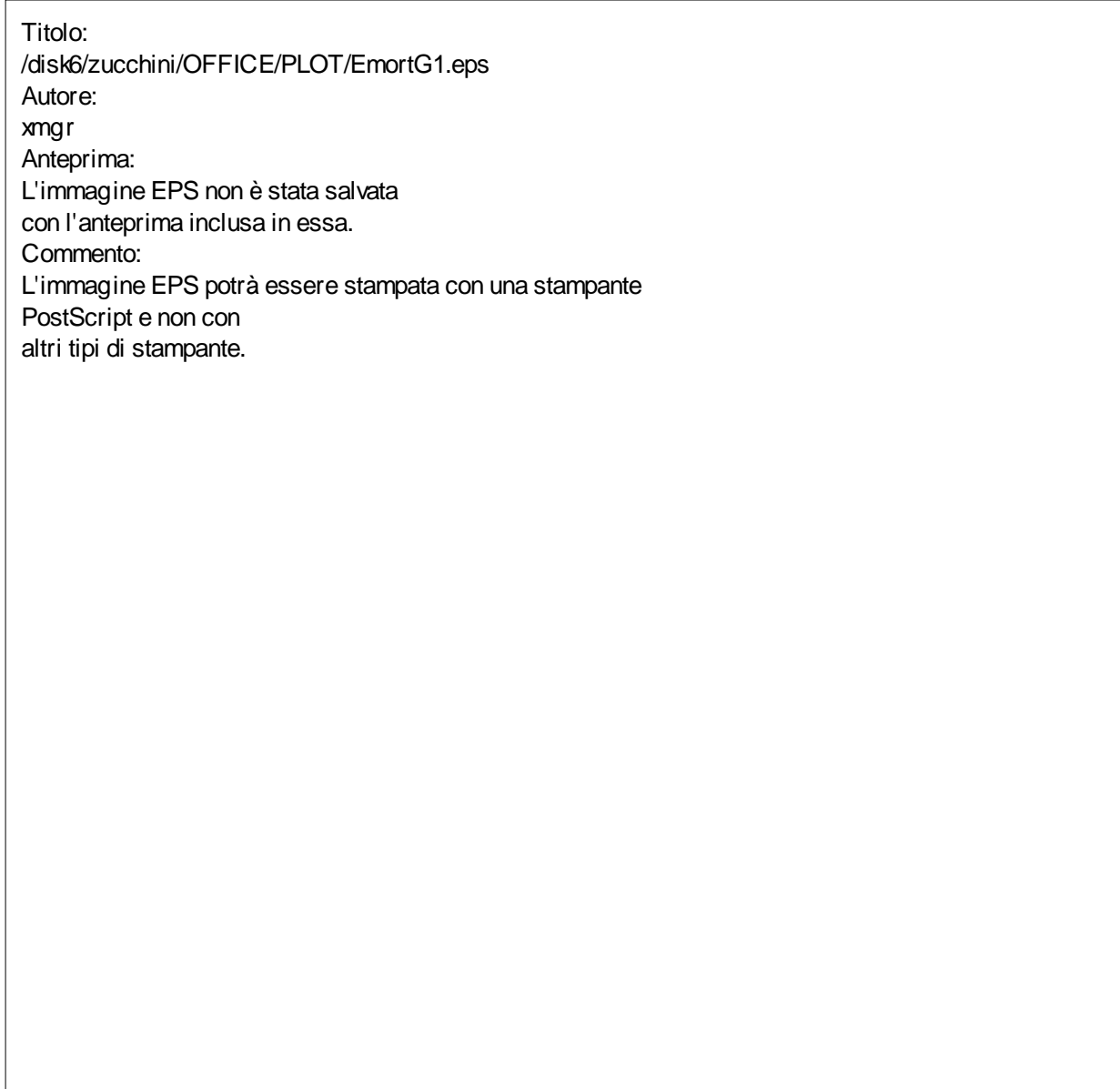

(c)

Fig. 13 - Comparison between the micro-mechanical model and FEA results for different stiffness ratios: (a) Young's moduli, (b) Poisson’s ratio and (c) Shear moduli 


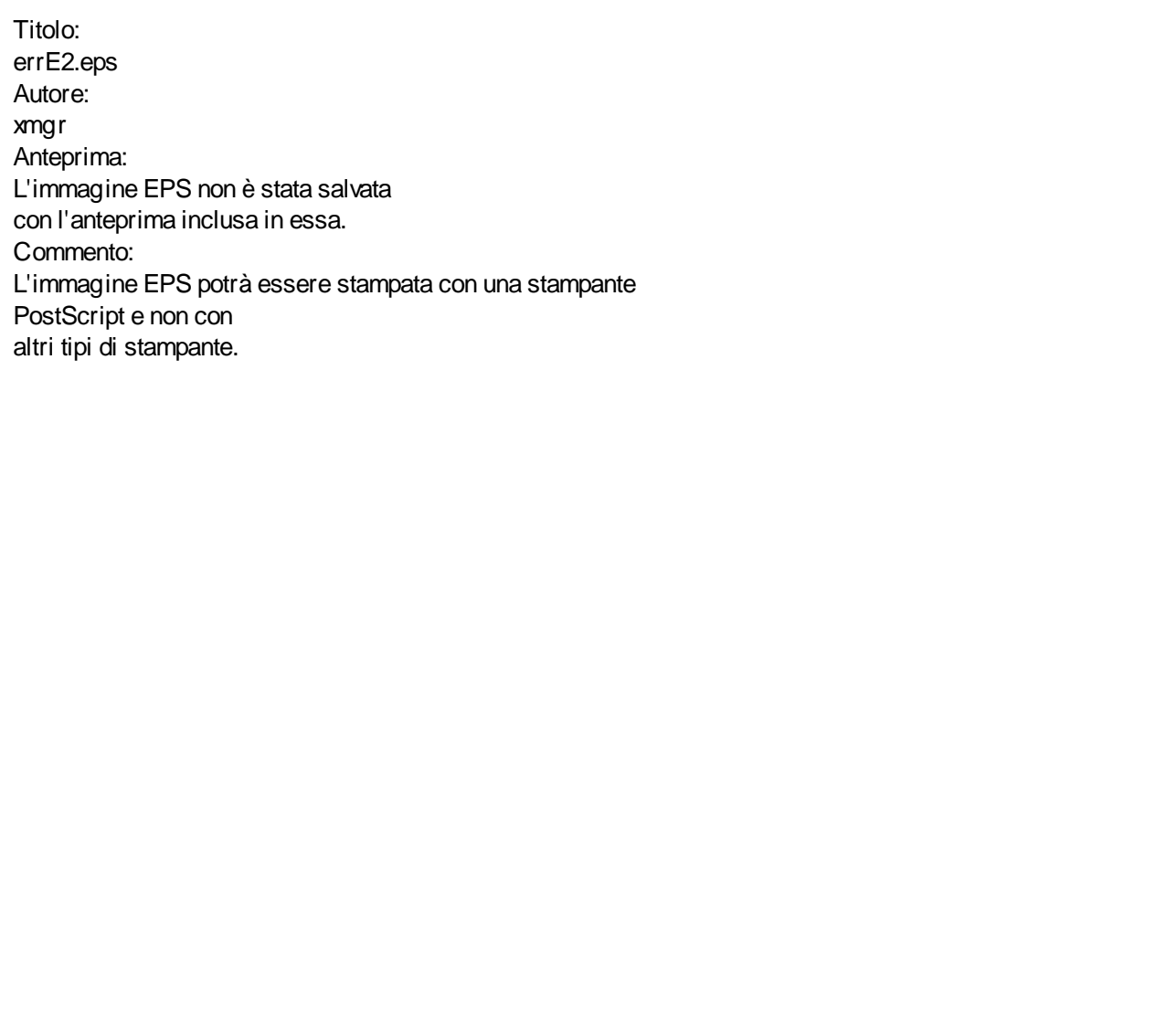




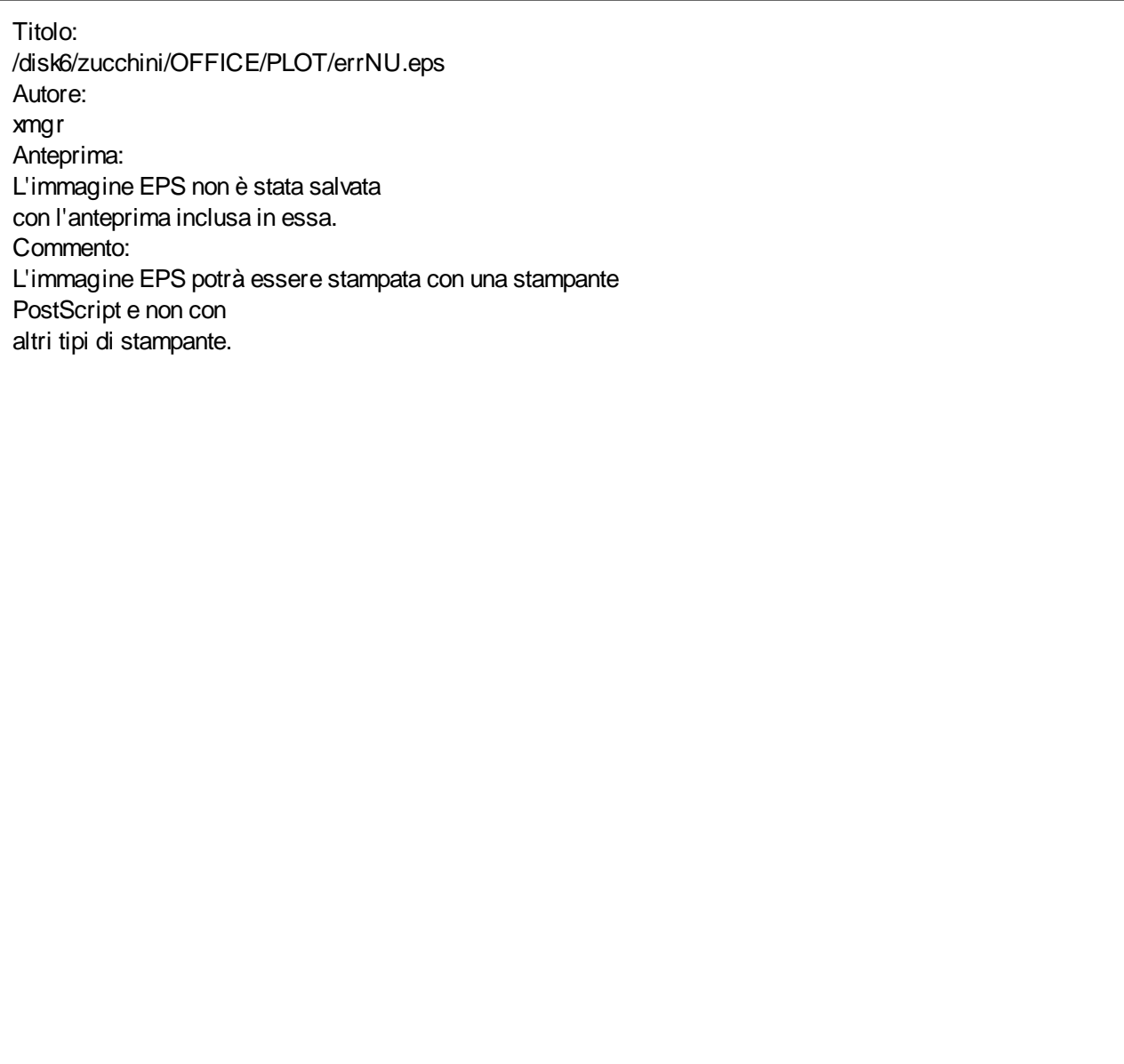

(b) 


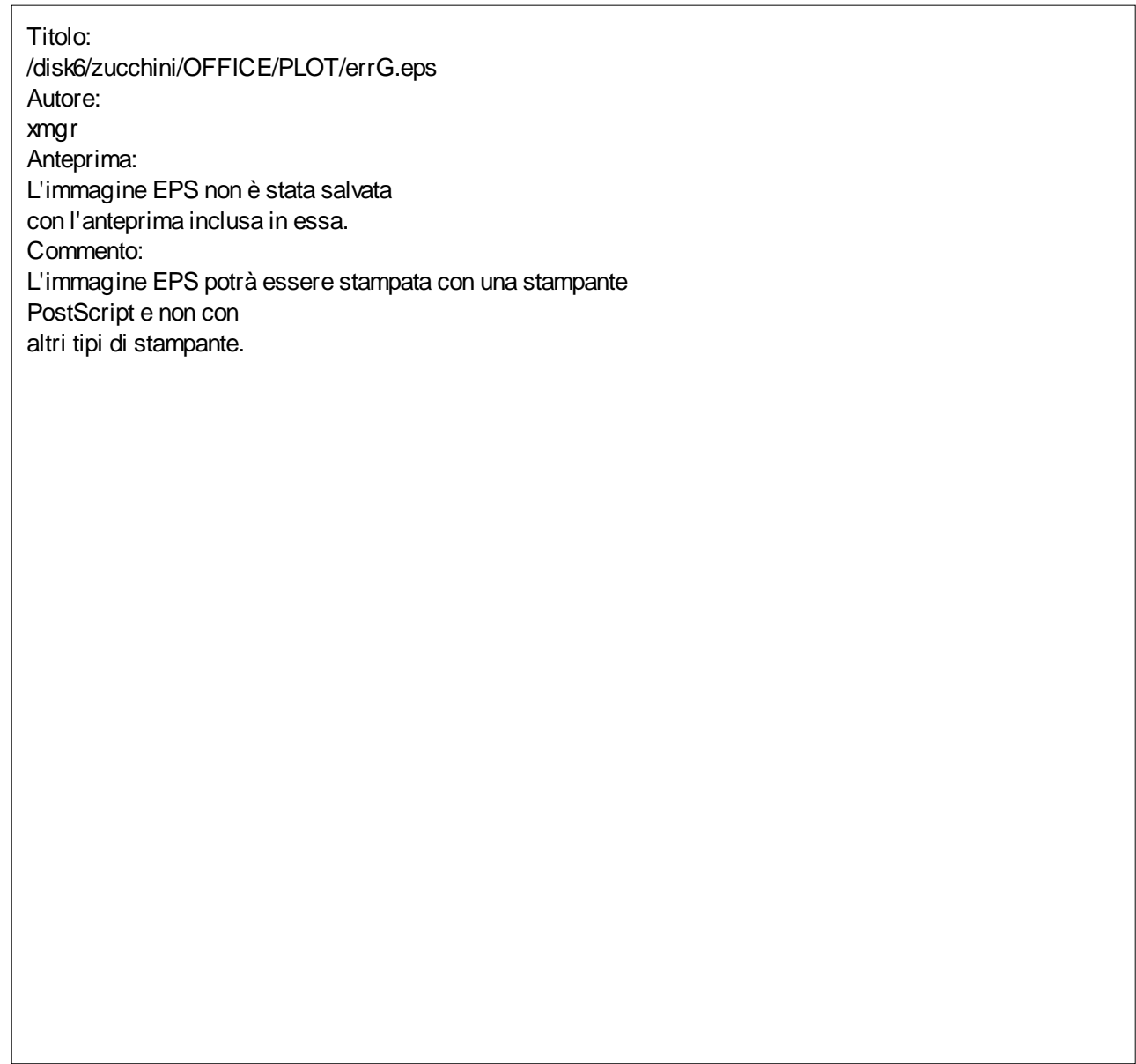

(c)

Fig. 14 - Comparison between the micro-mechanical model and FEA results for different stiffness ratios: (a) Young’s moduli, (b) Poisson’s ratio and (c) Shear moduli 


$$
\phi
$$


Titolo:

/usr/people/zucchini/OFFICE/MASONRY/fig12a.eps

Autore:

xmgr

Anteprima

L'immagine EPS non è stata salvata

con l'anteprima inclusa in essa.

Commento:

L'immagine EPS potrà essere stampata con una stampante

PostScript e non con

altri tipi di stampante. 


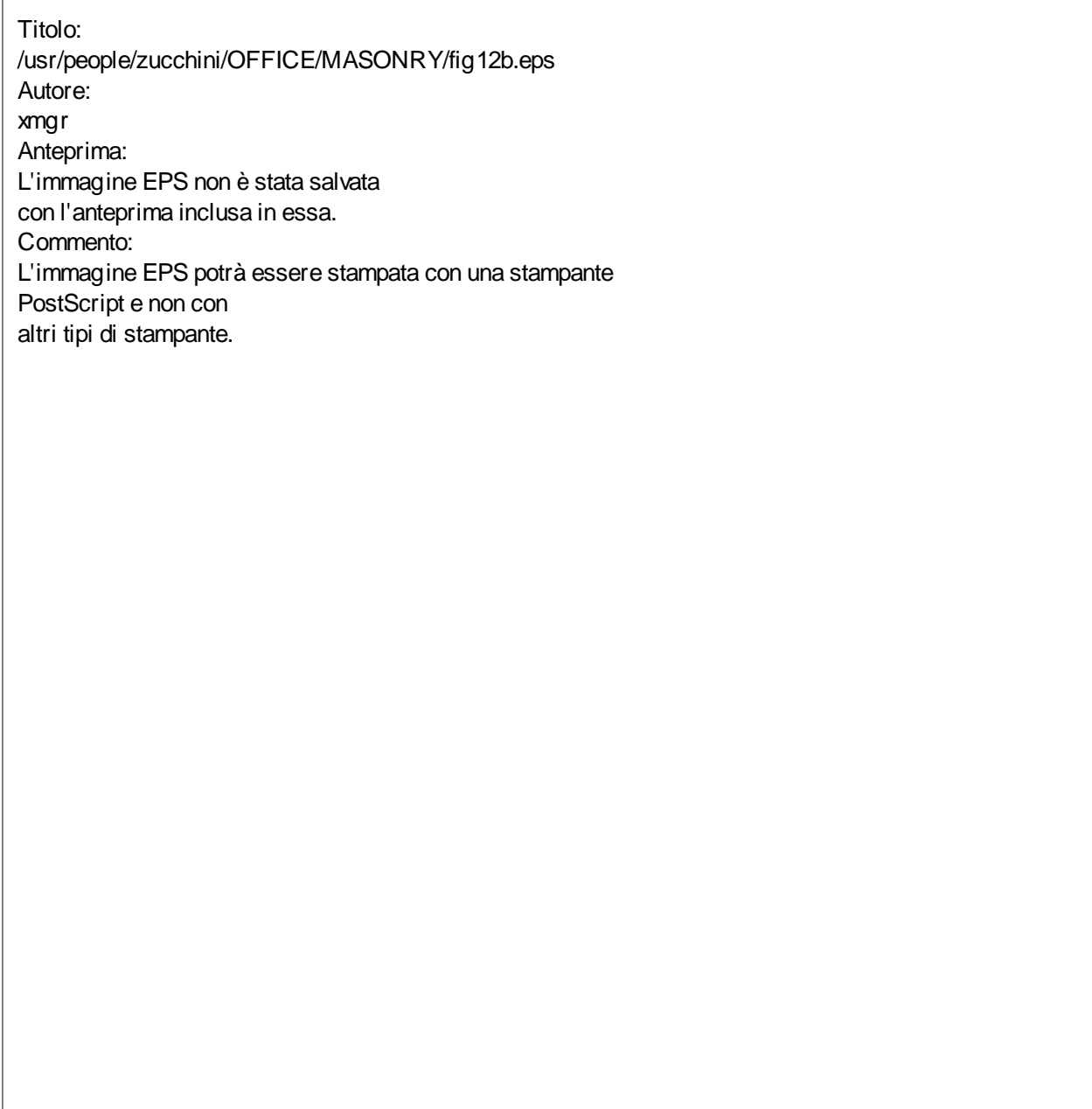

(b)

Fig. 16 - Calculated micro-mechanical failure criterion for masonry under biaxial in-plane loading (principal axes coincident with material axes):

(a) complete failure modes of the unit and mortar and (b) composite masonry failure. 


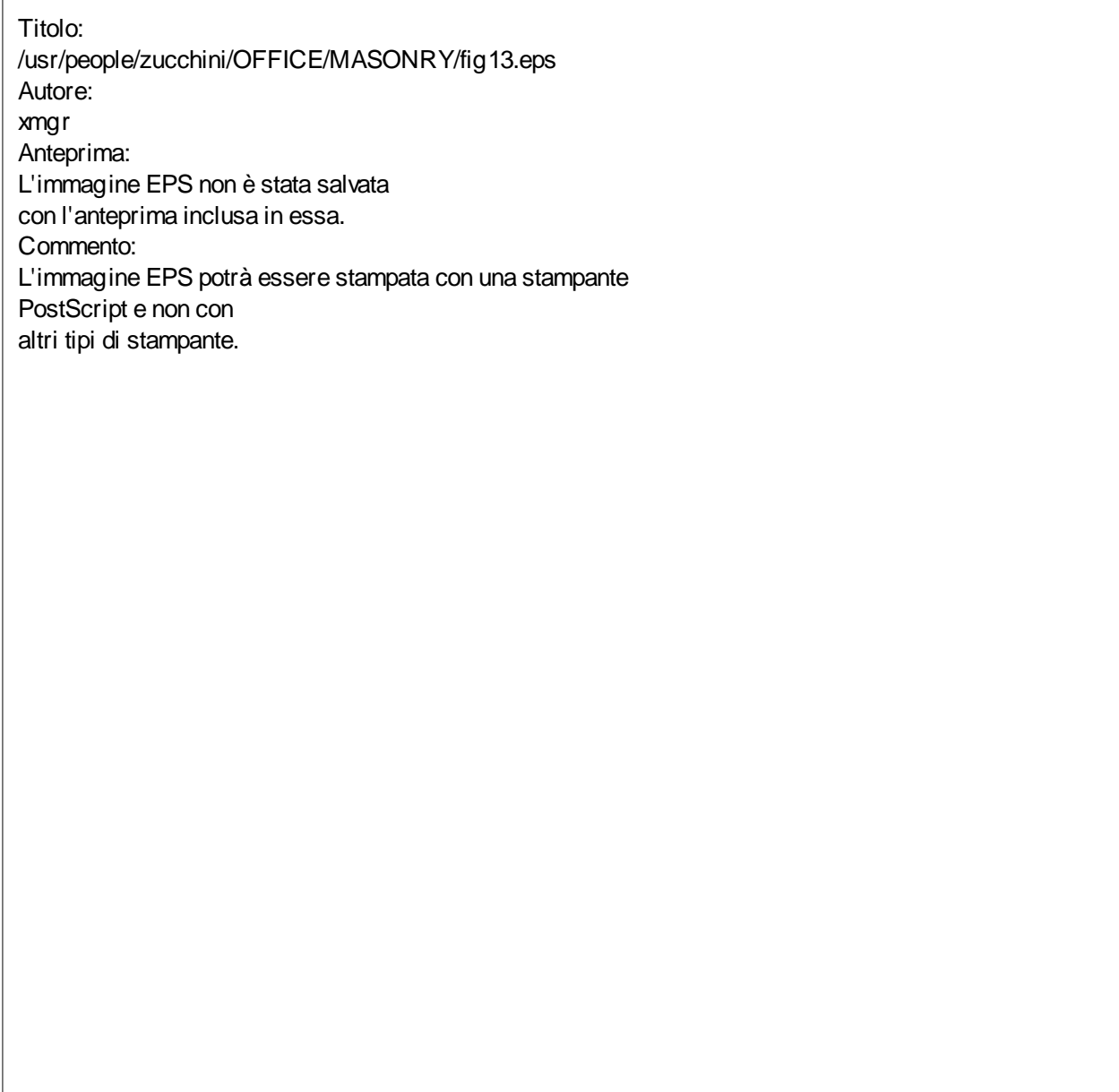

Fig. 17 - Comparison between micro-mechanical failure criterion and experimental results of Page (1981,1983). 